\title{
Gluing formulas for determinants of Dolbeault laplacians on Riemann surfaces
}

\author{
Richard A. WENTWORTh
}

\begin{abstract}
We present gluing formulas for zeta regularized determinants of Dolbeault laplacians on Riemann surfaces. These are expressed in terms of determinants of associated operators on surfaces with boundary satisfying local elliptic boundary conditions. The conditions are defined using the additional structure of a framing, or trivialization of the bundle near the boundary. An application to the computation of bosonization constants follows directly from these formulas.
\end{abstract}

\section{Introduction}

Given a conformal metric $\rho$ on a closed Riemann surface $M$ of genus $g$ and a hermitian metric $h$ on a holomorphic line bundle $L \rightarrow M$, let $\square_{L}=2 \bar{\partial}_{L}^{*} \bar{\partial}_{L}$ be the Dolbeault laplacian acting on sections of $L$. Determinants Det $\square_{L}$ are defined as the zeta regularized product of eigenvalues and are functions of $\rho, h$ and the moduli of $M$ and $L$ (see Section 2.4; in the case of a kernel the notation Det* $\square_{L}$ is used to emphasize that the zeta function is defined using only nonzero eigenvalues). In this paper, we derive gluing formulas for Det ${ }^{*} \square_{L}$ when $M$ is cut along closed curves, generalizing analogous identities obtained in $[10,16]$.

The main difference with the scalar case is an appropriate choice of boundary conditions for $\square_{L}$ on a surface with boundary. Local complex linear boundary conditions for the $\bar{\partial}$-complex do not exist, and it is common instead to impose spectral boundary conditions (for gluing formulas in this case, see, e.g.,[22] and the references therein). By contrast, in this paper we introduce local elliptic boundary conditions for sections of holomorphic bundles equipped with a framing, by which we mean a choice of trivialization near the boundary. These Alvarez boundary conditions are of mixed Dirichlet-Robin type and come from the splitting of sections $\Phi$ near the boundary into real and imaginary parts (denoted $\Phi^{\prime}$ and $\Phi^{\prime \prime}$ ) made possible by the framing. The conditions are similar to those studied by Alvarez in [1] for the case of traceless symmetric tensors - hence, the name - where there 
is a canonical choice of framing (see also [4, 9]). Because of the asymmetry, the boundary conditions are manifestly not complex linear. In particular, the $\bar{\partial}$-operator and the Dolbeault laplacian must be regarded as real operators $P_{L}$ and $D_{L}$, respectively (see Section 2.1). The Alvarez conditions on a section $\Phi$ are then $\left.\left(\Phi^{\prime \prime},\left(P_{L} \Phi\right)^{\prime \prime}\right)\right|_{\partial M}=0$. The advantage, however, is that the boundary value problem is compatible with a similar BVP on the adjoint bundle. This leads to an index theorem for $P_{L}$ and a generalization of the Polyakov-Alvarez formula for Det* $D_{L}$, which measures the variation under conformal changes of $(\rho, h)$ (see Theorems 2.1 and 2.2). Moreover, on a closed surface, Det ${ }^{*} D_{L}=\left(\text { Det }^{*} \square_{L}\right)^{2}$, so a gluing formula for $D_{L}$ provides one for $\square_{L}$ as well.

To state the main result, let $\Gamma \subset M$ be a collection of disjoint embedded oriented closed curves, and let $M_{\Gamma}$ denote the manifold with boundary obtained by cutting $M$ along $\Gamma$. Then a line bundle $L \rightarrow M$ pulls back to $M_{\Gamma}$ (we use the same notation $L$ ). There is a difference map $\delta_{\Gamma}$ on sections over $M_{\Gamma}$ which measures the difference of boundary values on each of the two components of $\partial M_{\Gamma}$ covering a component $\Gamma$. With this notation, we have the following.

Theorem 1.1. Given a framing of $L$ near $\Gamma$, let $\left\{\Phi_{i}\right\}$ (resp. $\left\{\Phi_{i}^{A}\right\}$ ) be a basis for $\operatorname{ker} D_{L}$ on $M$ (resp. for $\operatorname{ker} D_{L}^{A}$ on $M_{\Gamma}$ with Alvarez boundary conditions). Let $\operatorname{det}\left(\Phi_{i}, \Phi_{j}\right)$ denote the determinant in $(i, j)$ of the $L^{2}$-inner product on sections over $M$. Similarly for the sections $\Phi_{i}^{A}$ on $M_{\Gamma}$. Also $\operatorname{det}\left(\Phi_{i}, \Phi_{j}\right)_{\Gamma}$ denotes the determinant in $(i, j)$ of the $L^{2}$-inner product of restrictions of sections to $\Gamma$, and similarly for $\delta_{\Gamma} \Phi_{i}^{A}$. Assume the framing is generic in the sense of Definition 3.3. Then for any choice of $Q$, a self-adjoint elliptic positive pseudo-differential operator of order one on $\Gamma$, we have

$$
\left[\frac{\operatorname{Det}^{*} D_{L}}{\operatorname{det}\left(\Phi_{i}, \Phi_{j}\right)}\right]_{M}=c_{Q}\left[\frac{\operatorname{Det}^{*} D_{L}^{A}}{\operatorname{det}\left(\Phi_{i}^{A}, \Phi_{j}^{A}\right)}\right]_{M_{\Gamma}} \frac{\operatorname{det}\left(\delta_{\Gamma} \Phi_{i}^{A}, \delta_{\Gamma} \Phi_{j}^{A}\right)_{\Gamma}}{\operatorname{det}\left(\Phi_{i}^{\prime \prime}, \Phi_{j}^{\prime \prime}\right)_{\Gamma}} \operatorname{Det}_{Q}^{*} \mathcal{N}_{\Gamma},
$$

where $c_{Q}=2^{-\zeta_{Q}(0)}$ and $\mathcal{N}_{\Gamma}$ is a Neumann jump operator acting on sections over $\Gamma$ associated to the boundary value problem on $M_{\Gamma}$. See Section 3 for more details.

An important point is that because of the mixed boundary conditions, $\mathcal{N}_{\Gamma}$ is here an operator of order zero, rather than of order one as in the scalar case $[10,16]$. Following Friedlander-Guillemin [17], we define its determinant 
by choosing a regularizer $Q$ (see the definition (3.9)). Actually, in this case the dependence on the choice $Q$ is simply an overall constant $c_{Q}$.

In Section 4.1, we study the asymptotics of the determinant of the Neumann jump operator as $\Gamma$ shrinks to a point. For the scalar case, this was a key step in [33]. In a similar manner we find that the Neumann jump operator takes a standard form in the limit and that the asymptotic behavior of its determinant may be determined explicitly. As an application, the gluing formula can be used to cut and paste determinants for line bundles of different degrees. In particular, we give a new proof of a result on the behavior of determinants on exact sequences

$$
\left.0 \longrightarrow L \longrightarrow L(p) \longrightarrow L(p)\right|_{\{p\}} \longrightarrow 0
$$

when the line bundles are equipped with admissible metrics and $M$ with the Arakelov metric (see Section 4.2 for the definitions).

Theorem 1.2 (Insertion Theorem, $[5,8,11]$ ). Suppose $h^{1}(L)=\{0\}$, choose $p \in M$, and let $\mathcal{O}(p)$ be the line bundle determined by the divisor $\{p\}$. Let $\mathbb{1}_{p}$ be a nonzero holomorphic section of $\mathcal{O}(p)$ vanishing at $p$, and let $\hat{\omega}_{0}$ be a section of $L(p)=L \otimes \mathcal{O}(p)$ that is nonvanishing at $p$. Let $\left\{\omega_{i}\right\}_{i=1}^{m}$ be a basis of $H^{0}(L)$, and set $\hat{\omega}_{i}=\omega_{i} \otimes \mathbb{1}_{p}$, so that $\left\{\hat{\omega}_{i}\right\}_{i=0}^{m}$ is a basis for $H^{0}(L(p))$. Fix admissible metrics on $L$ and $\mathcal{O}(p)$ and the Arakelov metric on $M$, and let $L(p)$ have the induced metric. Then

$$
2 \pi\left\|\hat{\omega}_{0}(p)\right\|^{2} \frac{\operatorname{Det}^{*} \square_{L(p)}}{\operatorname{det}\left\langle\hat{\omega}_{i}, \hat{\omega}_{j}\right\rangle}=\frac{\operatorname{Det}^{*} \square_{L}}{\operatorname{det}\left\langle\omega_{i}, \omega_{j}\right\rangle},
$$

where $\langle\cdot, \cdot\rangle$ denotes the (hermitian) $L^{2}$-inner products on sections of $L$ and $L(p)$.

The equality above was proven in [11], and also in [8] (up to an overall constant) using the families index theorem. A higher dimensional version is proven in [5]. This formula is a key step in the proof of the bosonization formulas on Riemann surfaces which relate zeta-regularized determinants of Laplace operators acting on sections of line bundles to determinants of scalar laplacians (see [2, 6-8, 11, 13, 15, 28, 31], and for their role in string theory [12]). They are tantamount to a relationship between the metrics defined by Quillen and Faltings on the determinant of cohomology [14, 23]. For the definition of an admissible hermitian metric and of the Arakelov metric and Green's function $G(z, w)$ used below, see Section 4.2. Then in the notation of [15, Theorems 5.9 and 5.11], the result states that for $d \geq g-1$ 
and $M$ equipped with the Arakelov metric and associated Laplace-Beltrami operator $\Delta_{M}$, there are constants $c_{g}$ and $\delta_{g}$ depending only on the genus, and $\varepsilon_{g, d}$ depending only on the genus and degree (normalized so that $\varepsilon_{g, g-1}=1$ ), such that for any holomorphic line bundle $L$ of degree $d$ with admissible metric $h$ and associated divisor $[L]$ satisfying $h^{1}(L)=0$,

$$
\begin{aligned}
\frac{\operatorname{Det}^{*} \square_{L}}{\operatorname{det}\left\langle\omega_{i}, \omega_{j}\right\rangle}= & \varepsilon_{g, d} \delta_{g} \exp \left(c_{g} / 12\right)\left(\frac{\operatorname{Det}^{*} \Delta_{M}}{\operatorname{area}(M) \operatorname{det} \mathfrak{I m} \Omega}\right)^{-1 / 2} \\
& \times \frac{\prod_{i \neq j} G\left(p_{i}, p_{j}\right)}{\left\|\operatorname{det} \omega_{i}\left(p_{j}\right)\right\|^{2}}\|\vartheta\|^{2}\left([L]-\sum_{i=1}^{m} p_{i}-\delta, \Omega\right)
\end{aligned}
$$

where $m=d-g+1,\left\{p_{i}\right\}_{i=1}^{m}$ are generic points of $M,\left\{\omega_{i}\right\}_{i=1}^{m}$ is any basis for $H^{0}(M, L)$ and the pointwise and $L^{2}$-metrics are taken with respect to $h$. Here, $\Omega$ is the period matrix for a choice of homology basis, $\vartheta(Z, \Omega)$ the theta function and $\delta$ the Riemann divisor. We refer to [15] for the origin of these constants, and in particular the distinction between $c_{g}$ and $\delta_{g}$. The unknown constants appearing in (1.1) have been determined by Gillet-Soulé [19] and Soulé [29] and, using different methods, by Jorgenson [20] and in [33]. For example, the result of [33, Theorem 1.3] is

$$
c_{g}=-8 \log (2 \pi)+(g-1)\left(24 \zeta^{\prime}(-1)-1-2 \log \pi\right),
$$

where $\zeta(s)$ is the Riemann zeta function. The remaining values follow from Theorem 1.2. For completeness, we record the full result here. The following is a generalization of the genus 1 computation in [15, p. 117].

Corollary 1.1. Fay's constants $\delta_{g}$ and $\varepsilon_{g, d}$ defined in [15, Theorems 5.9 and 5.11] have values

$$
\begin{aligned}
\delta_{g} & =(2 \pi)^{g+1} \exp \left(c_{g} / 6\right), \\
\varepsilon_{g, d} & =(2 \pi)^{g-1-d}
\end{aligned}
$$

\section{The mixed boundary value problem}

\subsection{Real structures}

We begin with a construction that is completely elementary but will nevertheless serve to make precise the notions of a real operator and a real structure used in this paper. Let $V$ be a complex Hilbert space with hermitian inner product $\langle\cdot, \cdot\rangle$ and dual space $V^{*}$. Let $\mathcal{R}: V^{*} \rightarrow V$ be the complex 
antilinear isomorphism given by the Riesz representation: $f(a)=\langle a, \mathcal{R}(f)\rangle$, for all $a \in V, f \in V^{*}$. Note that the complex antilinear involution

$$
\imath: V \oplus V^{*} \longrightarrow V \oplus V^{*}:(a, f) \mapsto\left(\mathcal{R}(f), \mathcal{R}^{-1}(a)\right)
$$

satisfies $\left\langle\imath\left(a_{1}, f_{1}\right), \imath\left(a_{2}, f_{2}\right)\right\rangle=\overline{\left\langle\left(a_{1}, f_{1}\right),\left(a_{2}, f_{2}\right)\right\rangle}$ for the induced inner product on $V \oplus V^{*}$. Define

$$
V_{\mathbb{R}}=\operatorname{Fix}(\imath)=\left\{\left(a, \mathcal{R}^{-1}(a)\right): a \in V\right\}
$$

The map $\jmath: V \rightarrow V_{\mathbb{R}}: a \mapsto A=\left(a, \mathcal{R}^{-1}(a)\right)$ is then an $\mathbb{R}$-linear isomorphism. The real vector space $V_{\mathbb{R}}$ inherits a complete inner product $(\cdot, \cdot)$ from $V \oplus V^{*}$, and

$$
\left(\jmath a_{1}, \jmath a_{2}\right)=2 \mathfrak{R e}\left\langle a_{1}, a_{2}\right\rangle
$$

Let $T: V \rightarrow W$ be a (possibly unbounded) linear operator between complex Hilbert spaces. Then $\mathcal{R}^{-1} T \mathcal{R}: V^{*} \rightarrow W^{*}$ is also linear (with domain $\left.\mathcal{R}^{-1}(\operatorname{Dom} T)\right)$. The associated operator $\left(T, \mathcal{R}^{-1} T \mathcal{R}\right): V \oplus V^{*} \rightarrow W \oplus W^{*}$ commutes with the involution $\imath$ and hence induces a real linear map $P_{T}$ : $V_{\mathbb{R}} \rightarrow W_{\mathbb{R}}$ that makes the following diagram commute:

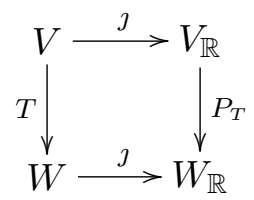

We call $P_{T}$ the real operator associated to $T$. Note that in the case $W=V$, it follows that the spectrum of $P_{T}: V_{\mathbb{R}} \rightarrow V_{\mathbb{R}}$ coincides with the real spectrum of $T: V \rightarrow V$ with twice the multiplicity: if $a \in V$ is nonzero with $T a=\lambda a$ and $\lambda \in \mathbb{R}$, then $\jmath a$ and $\jmath(i a)$ are independent eigenvectors of $P_{T}$, both with eigenvalue $\lambda$.

Finally, suppose that $V$ has a real structure. By this we mean a complex antilinear involution $\sigma: V \rightarrow V$ satisfying

$$
\left\langle\sigma a_{1}, \sigma a_{2}\right\rangle=\overline{\left\langle a_{1}, a_{2}\right\rangle}
$$

Then $\sigma_{\mathbb{R}}=\jmath \circ \sigma \circ \jmath^{-1}$ gives an involution of $V_{\mathbb{R}}$ which, by (2.2) and (2.3), is an isometry. Let $V_{\mathbb{R}}^{\prime}, V_{\mathbb{R}}^{\prime \prime}$ denote the $+1,-1$ eigenspaces of $\sigma_{\mathbb{R}}$, respectively. Then we have an orthogonal decomposition $V_{\mathbb{R}}=V_{\mathbb{R}}^{\prime} \oplus V_{\mathbb{R}}^{\prime \prime}$. For $A \in V_{\mathbb{R}}$, $A=A^{\prime}+A^{\prime \prime}$, where $A^{\prime}=(1 / 2)\left(A+\sigma_{\mathbb{R}} A\right), A^{\prime \prime}=(1 / 2)\left(A-\sigma_{\mathbb{R}} A\right)$. We refer 
to $A^{\prime}$ and $A^{\prime \prime}$ as the real and imaginary parts of $A$. There is a natural almost complex structure $J$ on $V_{\mathbb{R}}$ given by $J A=\jmath\left(i \jmath^{-1}(A)\right)$. A calculation shows that $\left(J A_{1}, J A_{2}\right)=\left(A_{1}, A_{2}\right)$, and $J\left(V_{\mathbb{R}}^{\prime}\right) \subset V_{\mathbb{R}}^{\prime \prime}, J\left(V_{\mathbb{R}}^{\prime \prime}\right) \subset V_{\mathbb{R}}^{\prime}$. As a consequence, if we define a symplectic structure on $V_{\mathbb{R}}$ by the pairing $\left(A_{1}, J A_{2}\right)$, then $V_{\mathbb{R}}^{\prime}$ and $V_{\mathbb{R}}^{\prime \prime}$ are lagrangian subspaces (i.e., maximal isotropic).

\subsection{Framed boundary conditions}

We apply the construction of Section 2.1 to sections of hermitian holomorphic line bundles on $M$. Let $M$ be a compact Riemann surface of genus $g$ with a (nonempty) boundary $\partial M$ and inclusion $\imath: \partial M \hookrightarrow M$. Without loss of generality, we may assume that $M$ is obtained from a closed Riemann surface by deleting finitely many disjoint coordinate disks. Each component of $\partial M$ has an open neighborhood in $M$ biholomorphic to an annulus $\left\{r_{1} \leq|z|<r_{2}\right\}$. We will refer to such a $z$ as an annular coordinate.

Let $L \rightarrow M$ be a holomorphic line bundle. A holomorphic structure on $L$ is equivalent to a Dolbeault operator $\bar{\partial}_{L}: \Omega^{0}(M, L) \rightarrow \Omega^{0,1}(M, L)$ satisfying the Leibniz rule. Equip $M$ with a conformal metric $\rho$ and $L$ with a hermitian metric $h$. The holomorphic and hermitian structures on $L$ give a unique unitary Chern connection $D=\left(\bar{\partial}_{L}, h\right)$, as well as an adjoint operator $\bar{\partial}_{L}^{*}$, and similarly on $L^{*}$. We will use the standard notation $h^{0}(L)=\operatorname{dim}_{\mathbb{C}} \operatorname{ker} \bar{\partial}_{L}$, $h^{1}(L)=\operatorname{dim}_{\mathbb{C}} \operatorname{coker} \bar{\partial}_{L}=\operatorname{dim}_{\mathbb{C}} \operatorname{ker} \bar{\partial}_{L}^{*}$.

There is a natural hermitian inner product on the space $\Omega^{0}(M, L)$ of smooth sections of $L$ given by

$$
\left\langle s_{1}, s_{2}\right\rangle_{M}=\int_{M} d A_{\rho}\left\langle s_{1}, s_{2}\right\rangle_{h},
$$

where $d A_{\rho}$ is the area form on $M$ coming from the metric $\rho$. The dual space is given by integration on $M: \Omega^{0}(M, L)^{*} \simeq \Omega^{1,1}\left(M, L^{*}\right)$. Then

$$
\Omega_{\mathbb{R}}^{0}(M, L) \subset \Omega^{0}(M, L) \oplus \Omega^{1,1}\left(M, L^{*}\right)
$$

is the real vector space constructed as in (2.1). Strictly speaking, here we should work with the $L^{2}$ and Sobolev completions. These are defined using the Chern connection $D$. Since this is standard, for notational simplicity we omit this from the notation.

We can also carry out this construction on $(0,1)$-forms:

$$
\Omega_{\mathbb{R}}^{0,1}(M, L) \subset \Omega^{0,1}(M, L) \oplus \Omega^{1,0}\left(M, L^{*}\right) .
$$


Denote the isomorphisms of real vector spaces

$$
\begin{gathered}
\jmath_{0}: \Omega^{0}(M, L) \longrightarrow \Omega_{\mathbb{R}}^{0}(M, L): \varphi \mapsto \Phi, \\
\jmath_{1}: \Omega^{0,1}(M, L) \longrightarrow \Omega_{\mathbb{R}}^{0,1}(M, L): \psi \mapsto \Psi
\end{gathered}
$$

or simply by $\jmath$ when the meaning is clear.

As in Section 2.1, define a (real, unbounded) linear operator $P_{L}: \Omega_{\mathbb{R}}^{0}$ $(M, L) \rightarrow \Omega_{\mathbb{R}}^{0,1}(M, L)$ making the following diagram commute:

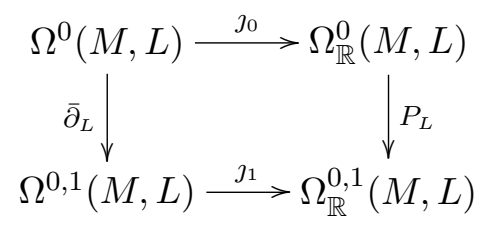

In terms of the decompositions (2.4) and (2.5), it follows that

$$
P_{L}=\left(\begin{array}{cc}
\bar{\partial}_{L} & 0 \\
0 & \left(\bar{\partial}_{L^{*}}\right)^{*}
\end{array}\right)
$$

Now consider the boundary. There is an hermitian inner product on $\Omega^{0}\left(\partial M, \imath^{*} L\right)$ given by

$$
\left\langle s_{1}, s_{2}\right\rangle_{\partial M}=\int_{\partial M} d s_{\rho}\left\langle s_{1}, s_{2}\right\rangle_{h},
$$

where $d s_{\rho}$ is the induced measure on $\partial M$. Note that $\partial M$ inherits an orientation from $M$ and the outward normal. Hence, integration gives an identification $\Omega^{0}\left(\partial M, \imath^{*} L\right)^{*}$ with $\Omega^{1}\left(\partial M, \imath^{*}\left(L^{*}\right)\right)$. With this understood, let

$$
\Omega_{\mathbb{R}}^{0}\left(\partial M, \imath^{*} L\right) \subset \Omega^{0}\left(\partial M, \imath^{*} L\right) \oplus \Omega^{1}\left(\partial M, \imath^{*}\left(L^{*}\right)\right)
$$

be the real vector space constructed as in the previous section.

The trace map

$$
\Omega^{0}(M, L) \longrightarrow \Omega^{0}\left(\partial M, \imath^{*} L\right):\left.\varphi \mapsto \varphi\right|_{\partial M}
$$

is induced by restriction. Using the Hodge star on $M$ to identify $\Omega^{1,1}\left(M, L^{*}\right)$ $\simeq \Omega^{0}\left(M, L^{*}\right)$, and on $\partial M$ to identify $\Omega^{1}\left(\partial M, \imath^{*} L^{*}\right) \simeq \Omega^{0}\left(\partial M, \imath^{*} L^{*}\right)$, there is 
a similar restriction map

$$
\Omega^{1,1}\left(M, L^{*}\right) \simeq \Omega^{0}\left(M, L^{*}\right) \longrightarrow \Omega^{0}\left(\partial M, \imath^{*} L^{*}\right) \simeq \Omega^{1}\left(\partial M, \imath^{*} L^{*}\right)
$$

The restriction maps combine to give a trace map $\Omega_{\mathbb{R}}^{0}(M, L) \rightarrow \Omega_{\mathbb{R}}^{0}\left(\partial M, \imath^{*} L\right)$. We carry out the same construction with $\Omega^{0,1}(M, L)$. Here, we define

$$
\Omega_{\mathbb{R}}^{1}\left(\partial M, \imath^{*} L\right) \subset \Omega^{1}\left(\partial M, \imath^{*} L\right) \oplus \Omega^{0}\left(\partial M, \imath^{*}\left(L^{*}\right)\right) .
$$

In this case, again using the Hodge star on $\partial M$ the trace map $\Omega_{\mathbb{R}}^{0,1}(M, L) \rightarrow$ $\Omega_{\mathbb{R}}^{1}\left(\partial M, \imath^{*} L\right)$ pulls-back the forms and restricts the section.

Definition 2.1. Let

$$
\mathfrak{B}\left(\partial M, \imath^{*} L\right)=\Omega_{\mathbb{R}}^{0}\left(\partial M, \imath^{*} L\right) \oplus \Omega_{\mathbb{R}}^{1}\left(\partial M, \imath^{*} L\right)
$$

be the space of boundary data. The trace map is the (real) linear map:

$$
\mathbf{b}_{\partial M}: \Omega_{\mathbb{R}}^{0}(M, L) \longrightarrow \mathfrak{B}\left(\partial M, \imath^{*} L\right):\left.\Phi \mapsto\left(\Phi, P_{L} \Phi\right)\right|_{\partial M}
$$

defined as above.

In order to define elliptic boundary conditions we will need real structures. These come from a choice of trivialization of $L$ near $\partial M$.

Definition 2.2. A framing of a holomorphic line bundle $L \rightarrow M$ is a trivialization (i.e., a nowhere vanishing holomorphic section) $\tau_{L}$ of $L$ near $\partial M$.

An important example of a framing is the following:

Example 2.1. Let $L$ be defined by a divisor $D$ compactly supported in $M$. Then by construction $L$ has a meromorphic section $\tau_{L}$ with zeros and poles exactly at $D$. In particular, $\tau_{L}$ gives a framing of $L$. While $\tau_{L}$ is only defined up to multiplication by a nonzero constant, we shall refer to any such choice as a canonical framing.

Given a framing and a section $\varphi$ of $L$ defined in a neighborhood of $\partial M$, write $\varphi=\left(\varphi^{\prime}+\mathrm{i} \varphi^{\prime \prime}\right) \tau_{L}$, where $\varphi^{\prime}, \varphi^{\prime \prime}$ are real valued functions. Then let $\sigma(\varphi)=\left(\varphi^{\prime}-\mathrm{i} \varphi^{\prime \prime}\right) \tau_{L}$. This defines a real structure on $\Omega^{0}\left(\partial M, \imath^{*} L\right)$. As in Section 2.1 , the boundary values of $\Phi \in \Omega_{\mathbb{R}}^{0}(M, L)$ therefore have real and imaginary parts $\Phi^{\prime}, \Phi^{\prime \prime}$. The framing also gives a real structure on boundary values of elements of $\Omega^{0,1}(M, L)$. Indeed, there is natural isomorphism $\left.T^{0,1} M\right|_{\partial M} \simeq$ 
$T(\partial M) \otimes \mathbb{C}$. Equivalently, the Hodge star gives a $\mathbb{C}$-linear isomorphism $*$ : $\Omega^{0}\left(\partial M, \imath^{*} L\right) \simeq \Omega^{1}\left(\partial M, \imath^{*} L\right)$ with $*^{2}=1$. If $\sigma_{0}$ is the real structure on $\Omega^{0}\left(\partial M, \imath^{*} L\right)$, then $\sigma_{1}=* \sigma_{0} *$ is a real structure on $\Omega^{1}\left(\partial M, \imath^{*} L\right)$. We let $\mathfrak{B}^{\prime}\left(\partial M, \imath^{*} L\right)\left(\operatorname{resp} . \mathfrak{B}^{\prime \prime}\left(\partial M, \imath^{*} L\right)\right)$ be the subspaces of $\mathfrak{B}\left(\partial M, \imath^{*} L\right)$ consisting of elements $\left(\Phi^{\prime}, \Psi^{\prime}\right)$ (resp. $\left.\left(\Phi^{\prime \prime}, \Psi^{\prime \prime}\right)\right)$.

- Note that there is a natural pairing of $\Omega_{\mathbb{R}}^{0}\left(\partial M, \imath^{*} L\right)$ and $\Omega_{\mathbb{R}}^{1}\left(\partial M, \imath^{*} L\right)$ defined as follows. If $\Phi=\jmath_{0}(\varphi), \Psi=\jmath_{1}(\psi)$ then

$$
(\Phi, \Psi)_{\partial M}=2 \mathfrak{R e} \int_{\partial M}\langle\varphi, \psi\rangle_{h} .
$$

- The real structure defines an almost complex structure on $\Omega_{\mathbb{R}}^{0}\left(\partial M, \imath^{*} L\right)$ and $\Omega_{\mathbb{R}}^{1}\left(\partial M, \imath^{*} L\right)$ as in Section 2.1. We extend this to an almost complex structure on the space of boundary values $\mathfrak{B}\left(\partial M, \imath^{*} L\right)$ by defining

$$
J_{\partial M}=\left(\begin{array}{cc}
0 & * J \\
* J & 0
\end{array}\right)
$$

(for simplicity, we will denote this operator simply by $J$ as well). This almost complex structure and the pairing (2.9) give a symplectic structure on $\mathfrak{B}\left(\partial M, \imath^{*} L\right)$ defined by $(f, J g)$. As in Section 2.1 , the subspaces $\mathfrak{B}^{\prime}\left(\partial M, \imath^{*} L\right)$ and $\mathfrak{B}^{\prime \prime}\left(\partial M, \imath^{*} L\right)$ are then lagrangian.

Definition 2.3. Let $\mathbf{b}_{\partial M}^{\prime}$ and $\mathbf{b}_{\partial M}^{\prime \prime}$ be the projections to the real and imaginary parts of $\mathbf{b}_{\partial M}$. We call the equation $\mathbf{b}_{\partial M}^{\prime}(\Phi)=0\left(\operatorname{resp} . \mathbf{b}_{\partial M}^{\prime \prime}(\Phi)=0\right)$ the real (resp. imaginary) Alvarez boundary conditions.

Note that $\mathbf{b}_{\partial M}^{\prime}$ and $\mathbf{b}_{\partial M}^{\prime \prime}$ take values in lagrangian subspaces of $\mathfrak{B}\left(\partial M, \imath^{*} L\right)$. We will use the same notation for the boundary map on $\Omega_{\mathbb{R}}^{0,1}(M, L) ;$ namely,

$$
\mathbf{b}_{\partial M}: \Omega_{\mathbb{R}}^{0,1}(M, L) \longrightarrow \mathfrak{B}\left(\partial M, \imath^{*} L\right):\left.\Psi \mapsto\left(P_{L}^{\dagger} \Psi, \Psi\right)\right|_{\partial M},
$$

where $P_{L}^{\dagger}$ is the formal adjoint of $P_{L}$. Then $\mathbf{b}_{\partial M}^{\prime}$ and $\mathbf{b}_{\partial M}^{\prime \prime}$ are defined similarly.

Since we here assume that $\partial M \neq \emptyset$, by a theorem of Grauert $L$ admits a global holomorphic trivialization $\mathbb{1}$ on $M$. Then $\tau_{L} / \mathbb{1}$ is a nowhere vanishing holomorphic function in a neighborhood of $\partial M$. We define the degree $\operatorname{deg}\left(\tau_{L}\right)$ of a framed line bundle to be the winding number of $\tau_{L} / \mathbb{1}$ (with 
the outward normal, summed over all components of $\partial M$ ). Clearly, the definition of degree is independent of the choice of trivialization $\mathbb{1}$. Note the following two important examples.

Example 2.2. (1) Let $s$ be a meromorphic section of $L$ satisfying imaginary Alvarez boundary conditions and with divisor $(s)$ compactly supported in the interior of $M$. Then $\operatorname{deg}\left(\tau_{L}\right)=\operatorname{deg}(s)$.

(2) Let $L=K^{q}$, where the framing is given by $\tau_{L}=(-\mathrm{i} d z / z)^{q}$ in local annular coordinates near $\partial M$. Then $\operatorname{deg}\left(\tau_{L}\right)=-q \chi(M)$. One can check that the real structure is independent of the choice of annular coordinate.

The Alvarez boundary conditions are of mixed Dirichlet-Robin type. Indeed, fix a framing $\tau_{L}$ of $L$, and let $h=\left\|\tau_{L}\right\|^{2}$. Then on $\partial M$, define

$$
\nu_{L, h}=-\frac{1}{2} \partial_{n} \log h
$$

where $n$ is the outward normal. Also, let $\Pi_{ \pm}=\frac{1}{2}\left(I \pm \sigma_{\mathbb{R}}\right)$ be the orthogonal projections to the real and imaginary parts. Then it is easy to see that $\mathbf{b}_{\partial M}^{\prime \prime}(\Phi)=0$ is equivalent to the conditions

$$
\begin{array}{r}
\left.\Pi_{-} \Phi\right|_{\partial M}=0 \\
\left.\left(\nabla_{n}+S\right) \Pi_{+} \Phi\right|_{\partial M}=0
\end{array}
$$

where $n$ is the outward normal, $\nabla$ is the induced connection on the bundle of real sections and $S=\nu_{L, h}$. Indeed, write $\varphi=\left(\varphi^{\prime}+\mathrm{i} \varphi^{\prime \prime}\right) \tau_{L}$. The Alvarez boundary conditions are $\varphi^{\prime \prime}=0$ and $\partial_{n} \varphi^{\prime}=0$ on $\partial M$. A local unitary frame is given by $\mathbf{e}_{L}=h^{-1 / 2} \tau_{L}$. Since the connection form in the frame $\mathbf{e}_{L}$ is purely imaginary, $\mathbf{e}_{L}$ is parallel with respect to $\nabla$ and the result follows from the expression $\Pi_{+} \Phi=\left(\varphi^{\prime} h^{1 / 2}\right) \mathbf{e}_{L}$.

\subsection{Heat kernels and an index theorem}

A straightforward calculation gives the following important integration by parts formula. For smooth sections $\Phi \in \Omega_{\mathbb{R}}^{0}(M, L)$ and $\Psi \in \Omega_{\mathbb{R}}^{0,1}(M, L)$,

$$
\left(P_{L} \Phi, \Psi\right)_{M}-\left(\Phi, P_{L}^{\dagger} \Psi\right)_{M}=\frac{1}{2}(\Phi, J \Psi)_{\partial M}
$$


where the pairing (2.9) appears on the right-hand side. Define the laplacian $D_{L}=2 P_{L}^{\dagger} P_{L}$ on smooth sections $\Omega_{\mathbb{R}}^{0}(M, L)$. Then from $(2.12)$ we have

$$
\begin{aligned}
\left(D_{L} \Phi_{1}, \Phi_{2}\right)_{M}-\left(\Phi_{1}, D_{L} \Phi_{2}\right)_{M} & =\left(\mathbf{b}_{\partial M}\left(\Phi_{1}\right), J \mathbf{b}_{\partial M}\left(\Phi_{2}\right)\right) \\
2\left(P_{L} \Phi_{1}, P_{L} \Phi_{2}\right)-\left(\Phi_{1}, D_{L} \Phi_{2}\right) & =\left[\left(\Phi_{1}^{\prime \prime}, J\left(P_{L} \Phi_{2}\right)^{\prime}\right)-\left(\left(P_{L} \Phi_{2}\right)^{\prime \prime}, J \Phi_{1}^{\prime}\right)\right] .
\end{aligned}
$$

Notice that the right-hand sides of (2.13) and (2.14) vanish identically for Alvarez boundary conditions. This gives positivity and formal self-adjointness of $D_{L}$. For the following result, see for example [18, Lemma 1.11.1].

Proposition 2.1. Assuming either real or imaginary Alvarez boundary conditions, the formal adjoint $P_{L}^{\dagger}$ extends to an unbounded operator on $\Omega_{\mathbb{R}}^{0,1}(M, L)$ as the the $L^{2}$-adjoint of $P_{L}$ on $\Omega_{\mathbb{R}}^{0}(M, L)$. Moreover, $D_{L}$ extends to an unbounded self-adjoint non-negative elliptic operator $D_{L}^{A}$ on sections $\Omega_{\mathbb{R}}^{0}(M, L)$ satisfying real (resp. imaginary) Alvarez boundary conditions. A similar statement holds for the laplacian $2 P_{L} P_{L}^{\dagger}$ on $\Omega_{\mathbb{R}}^{0,1}(M, L)$.

We now make a choice: henceforth, unless otherwise indicated, by Alvarez boundary conditions we will mean the condition $\mathbf{b}_{\partial M}^{\prime \prime}(\Phi)=0$. We write $D_{L}^{A}$ when we wish to emphasize that the laplacian $D_{L}$ is acting on the space of sections satisfying Alvarez boundary conditions.

Remark 2.1. By (2.14), $\operatorname{ker} D_{L}^{A} \subset \operatorname{ker} P_{L}$. Hence, $\operatorname{ker} D_{L}^{A}$ is real isomorphic to the space of holomorphic sections $\varphi$ of $L$ with local expression $\varphi=\varphi(z) \tau_{L}$ near $\partial M$, satisfying $\left.\mathfrak{I m}(\varphi(z))\right|_{\partial M}=0$.

Remark 2.2. If $\Phi$ is an eigensection of $D_{L}$ satisfying Alvarez boundary conditions with eigenvalue $\lambda \neq 0$, then $P_{L} \Phi$ is an eigensection of $2 P_{L} P_{L}^{\dagger}$ with the same eigenvalue $\lambda$, also satisfying Alvarez boundary conditions.

This simple observation is the raison d'être of the mixed boundary conditions we have chosen. By contrast, if $\varphi$ is an eigensection of $\square_{L}$ satisfying Dirichlet conditions, then $\bar{\partial}_{L} \varphi$ is a formal eigensection of $\bar{\partial}_{L} \bar{\partial}_{L}^{*}$, but does not necessarily satisfy an elliptic boundary condition. 
We also note the following

Proposition 2.2 (Serre duality). Fix a framing $\tau_{L}$ on $L \rightarrow M$. Then with respect to the duality

$$
\Omega^{0,1}(M, L) \simeq\left(\Omega^{0}\left(K \otimes L^{*}\right)\right)^{*}
$$

the framing on $K \otimes L^{*}$ is induced by that on $L$ and $-\mathrm{i} d z / z$, where $z$ is an annular coordinate near $\partial M$. In particular, with these Alvarez boundary conditions, coker $P_{L} \simeq \operatorname{ker} P_{L}^{\dagger} \simeq\left(\operatorname{ker} P_{K \otimes L^{*}}\right)^{\dagger}$.

Proof. The usual proof of Serre duality applies, modulo the boundary conditions. To understand these, choose a local annular coordinate $z$ near $\partial M$. Then with respect to the trivialization $\tau_{L}$, a smooth section $\psi d \bar{z} \in \Omega^{0,1}(M, L)$ satisfies Alvarez boundary conditions if $\mathfrak{I m}\left(\mathrm{i} \psi \mathrm{e}^{-\mathrm{i} \theta}\right)=0$ and $\mathfrak{I m}\left(\bar{\partial}_{L}^{*}(\psi d \bar{z})\right)=$ 0 on $\partial M$. The corresponding section of $\Omega^{0}\left(K \otimes L^{*}\right)$ is $\bar{\psi} h d z=\mathrm{i} z \bar{\psi} h(-\mathrm{i} d z / z)$, and so the Alvarez conditions are $\mathfrak{I m}(\mathrm{i} z \bar{\psi} h)=0$ and $\mathfrak{I m}\left(\partial_{\bar{z}}(\mathrm{i} z \bar{\psi} h) d \bar{z}\right)=0$ on $\partial M$. But on $\partial M, \mathfrak{I m}\left(\mathrm{i} \psi \mathrm{e}^{-\mathrm{i} \theta}\right)=0$ is equivalent to $\mathfrak{I m}(\mathrm{i} z \bar{\psi} h)=0$. In a similar way one shows that $\mathfrak{I m}\left(\partial_{\bar{z}}(\mathrm{i} z \bar{\psi} h) d \bar{z}\right)=-h \mathfrak{I m}\left(\bar{\partial}_{L}^{*}(\psi d \bar{z})\right)$. This proves the proposition.

In order to state a result for the small time expansion of the trace of the heat kernel, we will need the following quantities. Let $\Omega_{L, h}$ denote the Hermitian-Einstein tensor (cf.[21, IV.1.2]). In a local holomorphic frame we have

$$
\Omega_{L, h}=\mathrm{i} * F_{\left(\bar{\partial}_{L}, h\right)}=-\frac{1}{2} \Delta_{\rho} \log h
$$

where $F_{\left(\bar{\partial}_{L}, h\right)}$ is the curvature of the Chern connection. Note the following special case.

Lemma 2.1. Let $R_{\rho}$ and $\kappa_{\rho}$ denote the scalar and geodesic curvatures of $M$ and $\partial M$. With the hermitian metric on $K$ induced from the metric on $M, \Omega_{K, \rho^{-1}}=-(1 / 2) R_{\rho}$. For the framing $-\mathrm{i} d z / z, \nu_{K, \rho^{-1}}=\kappa_{\rho}$.

For the short time expansion of heat kernels, we refer to $[9,18]$. In particular, we use the result in [30, Section 5.3] and the expression for $S$ in (2.11).

Proposition 2.3. Let $L \rightarrow M$ be a holomorphic line bundle on $M$ with framing $\tau_{L}$. Let $\rho$ and $h$ be hermitian metrics on $M$ and L, respectively. 
Then for any function $f$, the trace with the heat kernel for the operator $D_{L}^{A}$ with Alvarez boundary conditions defined by $\tau_{L}$ has the following short time expansion:

$$
\begin{aligned}
\operatorname{Tr}\left(f \mathrm{e}^{-\varepsilon D_{L}^{A}}\right)= & \frac{1}{2 \pi \varepsilon} \int_{M} d A f+\frac{1}{12 \pi} \int_{M} d A f\left(6 \Omega_{L, h}+R_{\rho}\right) \\
& +\frac{1}{6 \pi} \int_{\partial M} d s f\left(\kappa_{\rho}-3 \nu_{L, h}\right)+O\left(\varepsilon^{1 / 2}\right)
\end{aligned}
$$

Theorem 2.1 (Index theorem). Let $L \rightarrow M$ be a holomorphic line bundle on $M$ with framing $\tau_{L}$. Then for Alvarez boundary conditions,

$$
\operatorname{index} P_{L}=\operatorname{dim}_{\mathbb{R}} \operatorname{ker} P_{L}-\operatorname{dim}_{\mathbb{R}} \operatorname{coker} P_{L}=2 \operatorname{deg}\left(\tau_{L}\right)+\chi(M) \text {. }
$$

Proof. From Proposition 2.3, Lemma 2.1, Remark 2.2 and Proposition 2.2

$$
\begin{aligned}
\operatorname{index} P_{L}= & \lim _{\varepsilon \rightarrow 0}\left\{\operatorname{Tr}\left(\mathrm{e}^{-2 \varepsilon P_{L}^{\dagger} P_{L}}\right)-\operatorname{Tr}\left(\mathrm{e}^{-2 \varepsilon P_{L} P_{L}^{\dagger}}\right)\right\} \\
= & \frac{1}{2 \pi} \int_{M} d A\left(\Omega_{L, h}-\Omega_{K L^{*},(\rho h)^{-1}}\right)-\frac{1}{2 \pi} \int_{\partial M} d s\left(\nu_{L, h}-\nu_{\left.K L^{*},(\rho h)^{-1}\right)}\right. \\
= & \frac{1}{2 \pi} \int_{M} d A 2 \Omega_{L, h}-\frac{1}{2 \pi} \int_{\partial M} d s 2 \nu_{L, h} \\
& +\frac{1}{4 \pi} \int_{M} d A R_{\rho}+\frac{1}{2 \pi} \int_{\partial M} d s \kappa_{\rho} .
\end{aligned}
$$

By the Gauss-Bonnet Theorem, the last two terms give the Euler characteristic $\chi(M)$. Write $\tau_{L}=f \mathbb{1}_{L}$, and let $h_{0}=\left\|\mathbb{1}_{L}\right\|^{2}$. Then near $\partial M, h=|f|^{2} h_{0}$ and

$$
\operatorname{deg}\left(\tau_{L}\right)=\frac{1}{2 \pi} \int_{\partial M} d s \partial_{n} \log |f| .
$$

On the other hand,

$$
\begin{aligned}
\frac{1}{2 \pi} \int_{M} d A \Omega_{L, h}-\frac{1}{2 \pi} \int_{\partial M} \nu_{L, h} & =-\frac{1}{4 \pi} \int_{M} d A \Delta \log h_{0}+\frac{1}{4 \pi} \int_{\partial M} d s \partial_{n} \log h \\
& =\frac{1}{4 \pi} \int_{\partial M} d s\left(-\partial_{n} \log h_{0}+\partial_{n} \log h\right) \\
& =\frac{1}{4 \pi} \int_{\partial M} d s \partial_{n} \log |f|^{2}=\operatorname{deg}\left(\tau_{L}\right) .
\end{aligned}
$$

The result follows.

Remark 2.3. By Example 2.2, if $K^{q}$ on $M$ is given the framing $(-\mathrm{i} d z / z)^{q}$ for annular coordinates at each component of $\partial M$, then $\operatorname{deg}\left(\tau_{K^{q}}\right)=-q \chi(M)$. 
Hence, by Theorem 2.1, index $P_{K^{q}}=(1-2 q) \chi(M)$. This agrees with [1, eq. (4.32)].

\subsection{Determinants of laplacians}

Following [24], we define determinants as follows. Suppose $M$ is closed with conformal metric $\rho$ and a hermitian holomorphic line bundle $L \rightarrow M$. Let $\left\{\lambda_{j}\right\}_{j=1}^{\infty}$ be the spectrum of $\square_{L}$ and form the zeta function $\zeta_{\square_{L}}(s)=$ $\sum_{\lambda_{j}>0} \lambda_{j}^{-s}$. Then $\zeta_{\square_{L}}(s)$ converges for $\mathfrak{R e}(s)$ sufficiently large, and by a theorem of Seeley [25] it is known that $\zeta_{\square_{L}}(s)$ is regular at $s=0$. Then $\log$ Det $^{*} \square_{L}:=-\zeta_{\square_{L}}^{\prime}(0)$. A similar definition applies to $\operatorname{Det}^{*} D_{L}$ on $M$, and to $\operatorname{Det}^{*} D_{L}^{A}$ when $M$ has boundary, $L$ has a framing, and we use Alvarez boundary conditions. When it is understood that the spectrum is strictly positive, we will omit the asterisk and write Det $\square_{L}$, etc.

When $M$ is closed, $D_{L}$ acting on $\Omega_{\mathbb{R}}^{0}(M, L)$ is the same as $\square_{L}$ acting on $\Omega(M, L)$, regarded as a real operator (see Section 2.1), and hence it has the same spectrum but with twice the multiplicity. Taking into account also the factor of 2 in the definition of the real inner product (see (2.2)), we have the following

Lemma 2.2. If $M$ is a closed Riemann surface with line bundle $L \rightarrow M$. Then for all $\lambda>0$,

$$
\operatorname{Det}\left(D_{L}+\lambda\right)=\left[\operatorname{Det}\left(\square_{L}+\lambda\right)\right]^{2}
$$

Similarly,

$$
\frac{\operatorname{Det}^{*} D_{L}}{\operatorname{det}\left(\Phi_{i}, \Phi_{j}\right)}=\left(2^{-h^{0}(L)} \frac{\operatorname{Det}^{*} \square_{L}}{\operatorname{det}\left\langle\omega_{i}, \omega_{j}\right\rangle}\right)^{2},
$$

where $\left\{\omega_{i}\right\}_{i=1}^{h^{0}(L)}$ is a basis (over $\mathbb{C}$ ) for $H^{0}(M, L)$ and $\left\{\Phi_{i}\right\}_{i=1}^{2 h^{0}(L)}$ is the associated basis (over $\mathbb{R}$ ) of $\operatorname{ker} D_{L}$ given by

$$
\Phi_{2 j}=\jmath\left(i \omega_{j}\right), \quad \Phi_{2 j-1}=\jmath\left(\omega_{j}\right)
$$

for $j=1, \ldots, h^{0}(L)$.

The main result of this section is the following

Theorem 2.2 (Polyakov-Alvarez formula). Let $\left\{\Phi_{i}\right\}_{i=1}^{m},\left\{\Psi_{j}\right\}_{j=1}^{n}$ be bases for ker $P_{L}$ and $\operatorname{ker} P_{L}^{\dagger}$, respectively, with Alvarez boundary conditions. Suppose 
the following relation for hermitian metrics: $\rho=\mathrm{e}^{2 \sigma} \hat{\rho}, h=\mathrm{e}^{2 f} \hat{h}$. Then

$$
\left[\frac{\operatorname{Det}^{*} D_{L}^{A}}{\operatorname{det}\left(\Phi_{i}, \Phi_{j}\right) \operatorname{det}\left(\Psi_{i}, \Psi_{j}\right)}\right]_{(\rho, h)}=\left[\frac{\operatorname{Det}^{*} D_{L}^{A}}{\operatorname{det}\left(\Phi_{i}, \Phi_{j}\right) \operatorname{det}\left(\Psi_{i}, \Psi_{j}\right)}\right]_{(\hat{\rho}, \hat{h})} \exp (S(\sigma, f))
$$

where

$$
\begin{aligned}
S(\sigma, f)=- & \frac{1}{6 \pi} \int_{M} d A_{\hat{\rho}}\left\{6 \nabla f \cdot \nabla(\sigma+f)+|\nabla \sigma|^{2}\right\} \\
& -\frac{1}{6 \pi} \int_{M} d A_{\hat{\rho}}\left\{6 \Omega_{L, \hat{h}}(\sigma+2 f)+R_{\hat{\rho}}(\sigma+3 f)\right\} \\
& +\frac{1}{3 \pi} \int_{\partial M} d s_{\hat{\rho}}\left\{3 \nu_{L, \hat{h}}(\sigma+2 f)-\kappa_{\hat{\rho}}(\sigma+3 f)\right\} .
\end{aligned}
$$

Proof. The argument follows [1]; here we only sketch the ideas. Let $\left\{\Phi_{j}\right\}$ be an orthonormal basis of eigensections of $D_{L}^{A}$ with eigenvalues $\lambda_{j}$. Then by Remark 2.2 , if $\Psi_{j}=\left(1 / \sqrt{\lambda_{j}}\right) P_{L} \Phi_{j}$, then $\left\{\Psi_{j}\right\}$ is an orthonormal basis of the subspace of eigensections of $2 P_{L} P_{L}^{\dagger}$ with nonzero eigenvalues and Alvarez boundary conditions. Let $\sigma=\sigma(t), f=f(t)$ be one parameter families of conformal deformations; $\dot{\sigma}$ and $\dot{f}$, their derivatives. One computes the variation of eigenvalues:

$$
\dot{\lambda}_{j}=-2 \lambda_{j}\left((\dot{\sigma}+\dot{f}) \Phi_{j}, \Phi_{j}\right)+2 \lambda_{j}\left(\dot{f} \Psi_{j}, \Psi_{j}\right)
$$

Then as in [1, pp. 148-149], the corresponding variation of the determinant is given by

$$
\begin{aligned}
\frac{d}{d t} & \log \operatorname{Det}^{*} D_{L}^{A} \\
& =f \cdot p \cdot \int_{\varepsilon}^{\infty} d t \sum_{\lambda_{j} \neq 0} \dot{\lambda}_{j} \mathrm{e}^{-t \lambda_{j}} \\
& =f \cdot p \cdot \int_{\varepsilon}^{\infty} d t \sum_{\lambda_{j} \neq 0}\left\{-2 \lambda_{j}\left((\dot{\sigma}+\dot{f}) \Phi_{j}, \Phi_{j}\right)+2 \lambda_{j}\left(\dot{f} \Psi_{j}, \Psi_{j}\right)\right\} \mathrm{e}^{-t \lambda_{j}} \\
& =-f \cdot p \cdot \int_{\varepsilon}^{\infty} d t \frac{d}{d t}\left\{-2 \operatorname{Tr}\left((\dot{\sigma}+\dot{f}) \mathrm{e}^{-2 t P_{L}^{\dagger} P_{L}}\right)+2 \operatorname{Tr}\left(\dot{f} \mathrm{e}^{\left.\left.-2 t P_{L} P_{L}^{\dagger}\right)\right\} .}\right.\right.
\end{aligned}
$$


Applying Proposition 2.3 to the heat kernel expansions for the laplacians on $D_{L}^{A}$ and $D_{K L^{*}}^{A}$,

$$
\begin{aligned}
& \frac{d}{d t} \log \operatorname{Det}^{*} D_{L}^{A} \\
& =-\frac{1}{6 \pi} \int_{M} d A_{\rho}\left(6 \Omega_{L, h}+R_{\rho}\right)(\dot{\sigma}+\dot{f})+\frac{1}{6 \pi} \int_{M} d A_{\rho}\left(6 \Omega_{K L^{*},(\rho h)^{-1}}+R_{\rho}\right) \dot{f} \\
& -\frac{1}{3 \pi} \int_{\partial M} d s_{\rho}\left(\kappa_{\rho}-3 \nu_{L, h}\right)(\dot{\sigma}+\dot{f})+\frac{1}{3 \pi} \int_{\partial M} d s_{\rho}\left(\kappa_{\rho}-3 \nu_{K L^{*},(\rho h)^{-1}}\right) \dot{f} .
\end{aligned}
$$

From Lemma 2.1 it follows that $\Omega_{K L^{*},(\rho h)^{-1}}=-(1 / 2) R_{\rho}-\Omega_{L, h}$, and $\nu_{K L^{*},(\rho h)^{-1}}=\kappa_{\rho}-\nu_{L, h}$. Hence,

$$
\begin{aligned}
\frac{d}{d t} \log \operatorname{Det}^{*} D_{L}^{A}= & -\frac{1}{6 \pi} \int_{M} d A_{\rho}\left\{6 \Omega_{L, h}(\dot{\sigma}+2 \dot{f})+R_{\rho}(\dot{\sigma}+3 \dot{f})\right\} \\
& -\frac{1}{3 \pi} \int_{\partial M} d s_{\rho}\left\{\kappa_{\rho}(\dot{\sigma}+3 \dot{f})-3 \nu_{L, h}(\dot{\sigma}+2 \dot{f})\right\}
\end{aligned}
$$

We have the following variations with respect to conformal changes:

$$
\begin{array}{r}
R_{\rho}=\mathrm{e}^{-2 \sigma}\left(R_{\hat{\rho}}-2 \Delta_{\hat{\rho}} \sigma\right), \quad \Omega_{L, h}=\mathrm{e}^{-2 \sigma}\left(\Omega_{L, \hat{h}}-\Delta_{\hat{\rho}} f\right), \\
\kappa_{\rho}=\mathrm{e}^{-\sigma}\left(\kappa_{\hat{\rho}}+\partial_{\hat{n}} \sigma\right), \quad \nu_{L, h}=\mathrm{e}^{-\sigma}\left(\nu_{L, \hat{h}}-\partial_{\hat{n}} f\right) .
\end{array}
$$

Plugging these into the above, the first term on the right-hand side of (2.19) becomes

$$
\begin{aligned}
& -\frac{1}{6 \pi} \int_{M} d A_{\hat{\rho}}\left\{6 \Omega_{L, \hat{h}}(\dot{\sigma}+2 \dot{f})+R_{\hat{\rho}}(\dot{\sigma}+3 \dot{f})\right\} \\
& \quad-\frac{1}{6 \pi} \int_{M} d A_{\hat{\rho}}\{6 \nabla f \cdot \nabla \dot{\sigma}+12 \nabla f \cdot \nabla \dot{f}+2 \nabla \sigma \cdot \nabla \dot{\sigma}+6 \nabla \sigma \cdot \nabla \dot{f}\} \\
& \quad+\frac{1}{6 \pi} \int_{\partial M} d s_{\hat{\rho}}\left\{12\left(\partial_{\hat{n}} f\right) \dot{f}+2\left(\partial_{\hat{n}} \sigma\right) \dot{\sigma}+6\left(\left(\partial_{\hat{n}} f\right) \dot{\sigma}+\left(\partial_{\hat{n}} \sigma\right) \dot{f}\right)\right\}
\end{aligned}
$$

whereas the second term on the right-hand side of (2.19) becomes

$$
\begin{aligned}
& -\frac{1}{3 \pi} \int_{\partial M} d s_{\hat{\rho}}\left\{\kappa_{\hat{\rho}}(\dot{\sigma}+3 \dot{f})-3 \nu_{L, \hat{h}}(\dot{\sigma}+2 \dot{f})\right\} \\
& -\frac{1}{3 \pi} \int_{\partial M} d s_{\hat{\rho}}\left\{\left(\partial_{\hat{n}} \sigma\right)(\dot{\sigma}+3 \dot{f})+3\left(\partial_{\hat{n}} f\right)(\dot{\sigma}+2 \dot{f})\right\} .
\end{aligned}
$$

The last terms on the right-hand sides of (2.20) and (2.21) cancel. The remaining terms can be integrated as in [1], giving the desired result. 
Remark 2.4. Consider the following special cases:

(1) $\partial M=\emptyset$. Then the formula in (2.18) coincides with the result in [15, Proposition 3.8]. Note that there is an overall factor of 2, from the fact that the determinant Det $^{*} D_{L}$, regarded as a real operator, is the square of the complex laplacian (see Lemma 2.2).

(2) If $L=K^{q}, h$ the induced metric from $M$, and $f=-q \sigma$, then (2.18) coincides with the result in [1, eq. (4.29)] (see Lemma 2.1).

(3) If $L$ is the trivial bundle $\mathcal{O}$ with the flat metric, then Alvarez boundary conditions amount to Dirichlet conditions on the real part and Neumann conditions on the imaginary part. Hence, the scalar determinant is $\operatorname{Det}^{*} D_{\mathcal{O}}^{A}=\left[\operatorname{Det}_{\text {neu. }}^{*}(\Delta)\right]\left[\operatorname{Det}_{\text {dir. }}(\Delta)\right]$.

(4) By Remark 2.2 and Serre duality Proposition 2.2 applied to the trivial bundle,

$$
\operatorname{Det}^{*} D_{K}^{A}=\operatorname{Det}^{*}\left(2 P_{\mathcal{O}} P_{\mathcal{O}}^{\dagger}\right)=\operatorname{Det}^{*}\left(2 P_{\mathcal{O}}^{\dagger} P_{\mathcal{O}}\right)=\operatorname{Det}^{*} D_{\mathcal{O}}^{A}
$$

\section{Factorization of determinants}

\subsection{The generalized Dirichlet-to-Neumann operator}

In this section we assume $M$ has non-empty boundary. Let $L \rightarrow M$ be a hermitian holomorphic bundle with framing $\tau_{L}$. The following is clear.

Lemma 3.1. The real and imaginary Alvarez boundary conditions are complimentary in the sense of [10, Definition 2.12].

Definition 3.1. The Poisson operator is characterized by the condition

$$
\mathcal{P}_{M}(\lambda): \mathfrak{B}^{\prime \prime}\left(\partial M, \imath^{*} L\right) \rightarrow \Omega_{\mathbb{R}}^{0}(M, L):(f, g) \mapsto \mathcal{P}_{M}(\lambda)(f, g)=\Phi,
$$

where $\Phi$ satisfies $\left(D_{L}+\lambda\right) \Phi=0$, and $\mathbf{b}_{\partial M}^{\prime \prime}(\Phi)=(f, g)$. The boundary operator is defined by

$$
\mathcal{A}_{M}(\lambda): \mathfrak{B}^{\prime \prime}\left(\partial M, \imath^{*} L\right) \rightarrow \mathfrak{B}^{\prime \prime}\left(\partial M, \imath^{*} L\right): \mathcal{A}_{M}(\lambda)=J \mathbf{b}_{\partial M}^{\prime} \mathcal{P}_{M}(\lambda)
$$

Hence, $\mathcal{A}_{M}(\lambda)$ is the analog of the Dirichlet-to-Neumann operator. Like the DN operator, $\mathcal{A}_{M}(\lambda)$ is elliptic and, by (2.13) it is self-adjoint. In this 
case, however, it is a zeroth-order pseudo-differential operator instead of first order.

In case $\lambda=0$, the Poisson, and hence also boundary operators are not necessarily everywhere defined nor are they a priori well defined. This can be seen from the integration by parts formula (2.13). The Poisson operator is defined at $(f, g)$ only if $(f, g)$ is orthogonal to the image by $J$ of boundary values of sections $\Phi \in \operatorname{ker} D_{L}$ satisfying imaginary Alvarez boundary conditions. Similarly, given any such $(f, g)$, the extension by the Poisson operator is only well defined up to addition of such $\Phi$. With this in mind, set

$$
\mathbb{A}_{M}^{\mathrm{alv}}=\left\{J \mathbf{b}_{\partial M}^{\prime}(\Phi): \Phi \in \operatorname{ker} D_{L}, \mathbf{b}_{\partial M}^{\prime \prime}(\Phi)=0\right\}
$$

Proposition 3.1. On the orthogonal complement of $\mathbb{A}_{M}^{\text {alv }}$, the family $\mathcal{A}_{M}(\lambda)$ extends continuously as $\lambda \rightarrow 0$ to an operator $\mathcal{A}_{M}(0)=\mathcal{A}_{M}$.

Proof. Let $\left\{\Phi_{i}^{A}\right\}_{i=1}^{\infty}$ be a complete set of eigensections for $D_{L}^{A}$ with eigenvalues $\left\{\lambda_{i}\right\}_{i=1}^{\infty}$, and $\lambda_{i}=0$ if and only if $i \leq n$. Choose a smooth extension map $E: \mathfrak{B}^{\prime \prime}\left(\partial M, \imath^{*} L\right) \rightarrow L^{2}(M)$ satisfying $\mathbf{b}_{\partial M}^{\prime \prime} E=I, \mathbf{b}_{\partial M}^{\prime} E=0$. To compute $\mathcal{P}_{M}(\lambda)(f, g)$ we need to solve the boundary value problem

$$
\left(D_{L}+\lambda\right) \Phi=0, \quad \mathbf{b}_{\partial M}^{\prime \prime}(\Phi)=(f, g)
$$

on $M$. From the definition of the extension, it suffices to solve

$$
\left(D_{L}+\lambda\right) \widetilde{\Phi}=-\left(D_{L}+\lambda\right) E(f, g), \quad \mathbf{b}_{\partial M}^{\prime \prime}(\widetilde{\Phi})=0
$$

for then $\Phi=E(f, g)+\widetilde{\Phi}$. Moreover, by the assumption on $E, J \mathbf{b}_{\partial M}^{\prime}(\widetilde{\Phi})=$ $\mathcal{A}_{M}(\lambda)(f, g)$. Now

$$
\begin{aligned}
\widetilde{\Phi}= & -\sum_{j=1}^{\infty} \frac{1}{\lambda_{j}+\lambda}\left(\left(D_{L}+\lambda\right) E(f, g), \Phi_{j}^{A}\right)_{M} \Phi_{j}^{A} \\
= & -\sum_{j=1}^{n}\left\{\frac{1}{\lambda}\left(D_{L} E(f, g), \Phi_{j}^{A}\right)_{M}+\left(E, \Phi_{j}\right)_{M}\right\} \Phi_{j}^{A} \\
& -\sum_{j=n+1}^{\infty} \frac{1}{\lambda_{j}+\lambda}\left(\left(D_{L}+\lambda\right) E(f, g), \Phi_{j}^{A}\right)_{M} \Phi_{j}^{A} .
\end{aligned}
$$


By (2.13), the first sum on the right-hand side reduces to (since $\left.\mathbf{b}_{\partial M}^{\prime \prime}\left(\Phi_{j}^{A}\right)=0\right)$ :

$$
\begin{aligned}
& =-\sum_{j=1}^{n}\left\{\frac{1}{\lambda}\left(\mathbf{b}_{\partial M}(E(f, g)), J \mathbf{b}_{\partial M}\left(\Phi_{j}^{A}\right)\right)+\left(E(f, g), \Phi_{j}^{A}\right)_{M}\right\} \Phi_{j}^{A} \\
& =-\sum_{j=1}^{n}\left\{\frac{1}{\lambda}\left(\mathbf{b}_{\partial M}^{\prime \prime}(E(f, g)), J \mathbf{b}_{\partial M}^{\prime}\left(\Phi_{j}^{A}\right)\right)+\left(E(f, g), \Phi_{j}^{A}\right)_{M}\right\} \Phi_{j}^{A} \\
& =-\sum_{j=1}^{n}\left\{\frac{1}{\lambda}\left((f, g), J \mathbf{b}_{\partial M}^{\prime}\left(\Phi_{j}^{A}\right)\right)+\left(E(f, g), \Phi_{j}^{A}\right)_{M}\right\} \Phi_{j}^{A} .
\end{aligned}
$$

Hence, if $(f, g) \in\left(\mathbb{A}_{M}^{\mathrm{alv}}\right)^{\perp}$

$$
\begin{aligned}
\mathcal{A}_{M}(\lambda)(f, g)= & -\sum_{j=1}^{n}\left(E(f, g), \Phi_{j}^{A}\right)_{M} J \mathbf{b}_{\partial M}^{\prime} \Phi_{j}^{A} \\
& -\sum_{j=n+1}^{\infty} \frac{1}{\lambda_{j}+\lambda}\left(\left(D_{L}+\lambda\right) E(f, g), \Phi_{j}^{A}\right)_{M} J \mathbf{b}_{\partial M}^{\prime} \Phi_{j}^{A} .
\end{aligned}
$$

This clearly extends continuously as $\lambda \rightarrow 0$, the second term gives the orthogonal projection to $\left(\mathbb{A}_{M}^{\text {alv }}\right)^{\perp}$.

Example 3.1. Consider the disk $B_{\varepsilon}$ of radius $\varepsilon$ with the euclidean metric and trivial line bundle, metric and framing. Then $\mathbb{A}_{B_{\varepsilon}}^{\text {alv }}=\{0\} \oplus \mathbb{R}$. By direct computation one shows that

$$
\mathcal{A}_{B_{\varepsilon}}(f, g)(\theta)=\sum_{n \neq 0}\left(\begin{array}{cc}
0 & -\mathrm{i} \sigma(n) \\
\mathrm{i} \sigma(n) & -\varepsilon /|n|
\end{array}\right)\left(\begin{array}{l}
\hat{f}(n) \\
\hat{g}(n)
\end{array}\right) \mathrm{e}^{\mathrm{i} n \theta}
$$

where

$$
f(\theta)=\sum_{n \in \mathbb{Z}} \hat{f}(n) \mathrm{e}^{\mathrm{i} n \theta}, \quad g(\theta)=\sum_{n \neq 0} \hat{g}(n) \mathrm{e}^{\mathrm{i} n \theta}
$$

and $\sigma(n)$ is the sign of $n$.

\subsection{The generalized Neumann jump operator}

Now suppose $M$ is closed. Let $\Gamma \subset M$ be a union of simple closed disjoint curves in $M$, and define $M_{\Gamma}$ to be the surface with boundary obtained 
from $M \backslash \Gamma$ by adjoining a double cover of $\Gamma$. We denote the connected components of $M_{\Gamma}$ by $R^{(i)}$, and by $g_{i}$ we mean the genus of $R^{(i)}$. Note that a conformal metric $\rho$ on $M$ induces one on $M_{\Gamma}$, and a holomorphic hermitian line bundle $L$ on determines one on $M_{\Gamma}$. In both cases, we use the same notation for the objects on $M$ and $M_{\Gamma}$.

Suppose that $\tau_{L}$ is a framing of $L \rightarrow M_{\Gamma}$. We will always assume such framings arise from local trivializations of $L$ in a neighborhood of $\Gamma \subset M$. We have the following:

Lemma 3.2. Let $d_{i}$ denote the degree of $L \rightarrow R^{(i)}$ defined by framing $\tau_{L}$, and let $d$ be the degree of $L \rightarrow M$. Then $d=\sum_{i} d_{i}$.

Proof. Let $s$ be a meromorphic section of $L$ with no zeros or poles on $\Gamma$, and let $s_{i}$ denote the induced meromorphic sections of $L \rightarrow R^{(i)}$. Clearly, $d=\operatorname{deg}(s)=\sum_{i} \operatorname{deg}\left(s_{i}\right)$. Write $\tau_{L}=f s$ for a nowhere vanishing function $f$ defined in a neighborhood of $\Gamma$. Then the local winding number of $\tau_{L}$ is the sum of local winding numbers of $f$ and $s$. On the other hand, for each component of $\Gamma$, the local winding numbers of $f$ on the two copies in $M_{\Gamma}$ cancel, since they are defined in terms of outward normals. Hence,

$$
\sum_{i=1} \operatorname{deg}\left(\tau_{L}\right)_{R^{(i)}}=\sum_{i=1} \operatorname{deg}\left(s_{i}\right)=d .
$$

The additivity of the Euler characteristic and Theorem 2.1 imply

Corollary 3.1. Let $M$ be a closed surface and $\Gamma \subset M$ a union of simple closed curves dividing $M$ into surfaces $R^{(i)}, i=1, \ldots, \ell$, with boundary. Let $P_{L}$ be the real operator associated to $\bar{\partial}_{L}$ on $\Omega^{0}(M, L)$, and on $\Omega_{\mathbb{R}}^{0}\left(R^{(i)}, L\right)$ with Alvarez boundary conditions defined by a framing $\tau_{L}$. Then

$$
\operatorname{index}\left(P_{L}\right)=\sum_{i=1}^{\ell} \operatorname{index}\left(P_{L}\right)_{R^{(i)}} .
$$

Choose an orientation for $\Gamma$. We define maps

$$
\mathbf{b}_{\Gamma}: \Omega_{\mathbb{R}}^{0}(M, L) \rightarrow \mathfrak{B}\left(\Gamma, \imath^{*} L\right):=\Omega_{\mathbb{R}}^{0}\left(\Gamma, \imath^{*} L\right) \oplus \Omega_{\mathbb{R}}^{1}\left(\Gamma, \imath^{*} L\right)
$$

(and $\mathbf{b}_{\Gamma}^{\prime}, \mathbf{b}_{\Gamma}^{\prime \prime}$ ) by restriction. The double cover $\partial M_{\Gamma} \rightarrow \Gamma$ gives a diagonal and difference map

$$
\begin{aligned}
& \imath_{\Delta}: \mathfrak{B}\left(\Gamma, \imath^{*} L\right) \longrightarrow \mathfrak{B}\left(\partial M_{\Gamma}, \imath^{*} L\right), \\
& \delta_{\Gamma}: \mathfrak{B}\left(\partial M_{\Gamma}, \imath^{*} L\right) \longrightarrow \mathfrak{B}\left(\Gamma, \imath^{*} L\right) .
\end{aligned}
$$


The maps $\imath_{\Delta}$ and $\delta_{\Gamma}$ depend on the choice of orientation of $\Gamma$. We assume that such an orientation has been fixed once and for all.

We now come to the following crucial definition:

Definition 3.2. The Neumann jump operator $\mathcal{N}_{\Gamma}(\lambda): \mathfrak{B}^{\prime \prime}\left(\Gamma, \imath^{*} L\right) \longrightarrow$ $\mathfrak{B}^{\prime \prime}\left(\Gamma, \imath^{*} L\right)$ is defined by the composition: $\mathcal{N}_{\Gamma}(\lambda)(f, g)=\delta_{\Gamma} \mathcal{A}_{M_{\Gamma}}(\lambda)\left(\imath_{\Delta}(f, g)\right)$.

Then $\mathcal{N}_{\Gamma}(\lambda)$ is a self-adjoint elliptic pseudo-differential operator of order zero. Note that $\mathcal{N}_{\Gamma}(\lambda)$ is invertible for all $\lambda>0$, since the kernel would be the boundary value of a global section in the kernel of $D_{L}+\lambda$. A calculation similar to the one in [10, Proposition 4.4] leads to the following:

Proposition 3.2. Choose coordinates with $\rho \equiv 1$ on $\Gamma$ and an appropriate gauge so that the unitary frame associated to $\tau_{L}$ is parallel along $\Gamma$. Then the symbol of $\mathcal{N}_{\Gamma}(\lambda)$ is given by

$$
\sigma_{\mathcal{N}_{\Gamma}(\lambda)}(x, \xi)=2\left(I+r_{\lambda}(x, \xi)\right) a_{\lambda}(\xi)
$$

where $a_{\lambda}(\xi)$ is a block diagonal with respect to the components of $\Gamma$, with blocks equal to

$$
\frac{1}{\left(\xi^{2}+\lambda\right)^{1 / 2}}\left(\begin{array}{cc}
\lambda / 2 & -\mathrm{i} \xi \\
\mathrm{i} \xi & -2
\end{array}\right)
$$

and $r_{\lambda}(x, \xi)$ is a matrix symbol with parameter (cf.[26, Definition 9.1]) satisfying

$$
\left\|\partial_{x}^{m} \partial_{\xi}^{n} r_{\lambda}(x, \xi)\right\| \leq C_{m, n}\left(1+|\xi|+|\lambda|^{1 / 2}\right)^{-2-n}
$$

for all $m, n \geq 0$. The same estimate holds for $\dot{r}_{\lambda}(x, \xi)=d r_{\lambda}(x, \xi) / d \lambda$.

Let

$$
\star: \mathfrak{B}^{\prime \prime}\left(\Gamma, \imath^{*} L\right) \rightarrow \mathfrak{B}^{\prime \prime}\left(\Gamma, \imath^{*} L\right):(f, g) \mapsto(* g, * f)
$$

Corollary 3.2. For $\lambda>0$ we have $\mathcal{N}_{\Gamma}(\lambda)=2(I+R(\lambda)) A(\lambda)$, where

(1) $A(\lambda)$ is an invertible elliptic pseudo-differential operator of order zero satisfying

$$
\star A(\lambda)=-A(\lambda)^{-1} \star .
$$

(2) $R(\lambda)$ is a pseudo-differential operator with parameter of order -2 with uniform bound $O\left(\lambda^{-1}\right)$. 
Proof. Define

$$
\begin{aligned}
& A(\lambda)=\left(\square_{\Gamma}+\lambda\right)^{-1 / 2}\left(\begin{array}{cc}
\lambda / 2 & * \nabla_{\Gamma^{*}} \\
-\nabla_{\Gamma} & -2
\end{array}\right), \\
& R(\lambda)=\frac{1}{2} \mathcal{N}_{\Gamma}(\lambda) A(\lambda)^{-1}-I
\end{aligned}
$$

acting on $\mathfrak{B}^{\prime \prime}\left(\Gamma, \imath^{*} L\right)$, where the covariant derivatives and laplacian are with respect to the metric on $\partial M$ induced by $\rho$ and the Chern connection. Then (1) is clear from the definition, and (2) follows from Proposition 3.2 and [26, Corollary 9.1].

As with the boundary operator, the jump operator is not everywhere defined for $\lambda=0$. In order to rectify this, let $\mathbb{A}_{\Gamma}=\mathbb{A}_{\Gamma}^{\mathrm{ker}} \oplus \mathbb{A}_{\Gamma}^{\text {alv }}$, where

$$
\begin{aligned}
& \mathbb{A}_{\Gamma}^{\mathrm{ker}}=\left\{\mathbf{b}_{\Gamma}^{\prime \prime}(\Phi): \Phi \in \operatorname{ker} D_{L} \subset \Omega_{\mathbb{R}}^{0}(M, L)\right\}, \\
& \mathbb{A}_{\Gamma}^{\mathrm{alv}}=\left\{\delta_{\Gamma} J\left(\mathbf{b}_{\partial M_{\Gamma}}^{\prime}(\Phi)\right): \Phi \in \operatorname{ker} D_{L} \subset \Omega_{\mathbb{R}}^{0}\left(M_{\Gamma}, L\right), \mathbf{b}_{\partial M_{\Gamma}}^{\prime \prime}(\Phi)=0\right\} .
\end{aligned}
$$

Notice that $\mathbb{A}_{\Gamma}^{\mathrm{ker}} \subset \Omega_{\mathbb{R}}^{0}\left(\Gamma, \imath^{*} L\right) \oplus\{0\}, \mathbb{A}_{\Gamma}^{\text {alv }} \subset\{0\} \oplus \Omega_{\mathbb{R}}^{1}\left(\Gamma, \imath^{*} L\right)$. In particular, $\mathbb{A}_{\Gamma}^{\text {ker }} \perp \mathbb{A}_{\Gamma}^{\text {alv }}$. Now Propositions 3.1 and 3.2 imply

Proposition 3.3. On the orthogonal complement of $\mathbb{A}_{\Gamma}$, the family $\mathcal{N}_{\Gamma}(\lambda)$ extends continuously as $\lambda \rightarrow 0$ to a zeroth-order operator $\mathcal{N}_{\Gamma}(0)=\mathcal{N}_{\Gamma}$.

We also record the following:

Lemma 3.3. Assume coker $P_{L}=\{0\}$ on $M$ and on $M_{\Gamma}$. Then $\operatorname{dim}_{\mathbb{R}} \mathbb{A}_{\Gamma}^{\mathrm{ker}}=$ $\operatorname{dim}_{\mathbb{R}} \mathbb{A}_{\Gamma}^{\text {alv }}$.

Proof. Let $V=\left\{\mathbf{b}_{\Gamma}^{\prime}(\Phi): \Phi \in \operatorname{ker} D_{L}, \mathbf{b}_{\Gamma}^{\prime \prime}(\Phi)=0\right\}$. Then since any holomorphic section vanishing on $\Gamma$ must vanish identically, we have by the assumption on cokernels Corollary 3.1,

$$
\operatorname{dim}_{\mathbb{R}} \mathbb{A}_{\Gamma}^{\mathrm{ker}}=2 h^{0}(L)-\operatorname{dim}_{\mathbb{R}} V=\operatorname{dim}_{\mathbb{R}} \operatorname{ker} D_{L}^{A}-\operatorname{dim}_{\mathbb{R}} V
$$

On the other hand, consider the surjective map ker $D_{L}^{A} \rightarrow \mathbb{A}_{\Gamma}^{\mathrm{alv}}$. Any element in the kernel corresponds to a global holomorphic section satisfying the extra condition $\mathbf{b}_{\Gamma}^{\prime \prime}(\Phi)=0$. Hence,

$$
\operatorname{dim}_{\mathbb{R}} \operatorname{ker} D_{L}^{A}-\operatorname{dim}_{\mathbb{R}} V=\operatorname{dim}_{\mathbb{R}} \mathbb{A}_{\Gamma}^{\text {alv }}
$$

and the result follows. 


\subsection{Determinants of zeroth-order operators}

Let $T$ be a positive self-adjoint elliptic pseudo-differential operator of order zero on the real Hilbert space $L^{2}\left(S^{1}\right) \oplus L^{2}\left(S^{1}\right)$ (where the $L^{2}$ functions are real valued). The usual zeta regularization procedure does not apply to $T$. In order to define its determinant, we need to choose a regularizer. By this we mean a positive self-adjoint elliptic pseudo-differential operator $Q$ of order 1 on $L^{2}\left(S^{1}\right)$. Given $Q$, we extend it diagonally on $L^{2}\left(S^{1}\right) \oplus L^{2}\left(S^{1}\right)$ and denote this extended operator also by $Q$.

Next, define $\log T$ as follows. Let $\gamma \subset \mathbb{C} \backslash\{\mathfrak{R e} z \leq 0\}$ be a closed curve containing the spectrum of $T$. Then define

$$
\log T=\frac{1}{2 \pi \mathrm{i}} \int_{\gamma} d z(\log z)(z-T)^{-1}
$$

where $\log$ is the branch of the logarithm on $\mathbb{C} \backslash\{\mathfrak{R e} z \leq 0\}$ with $-\pi<$ $\arg \log z<\pi$. Then following [17], we set

$$
\log \operatorname{Det}_{Q} T=\left.f p \operatorname{Tr}\left(Q^{-s} \log T\right)\right|_{s=0}
$$

While this definition of the determinant depends on $Q$, it is nevertheless very suitable for our purposes. The main properties that we need are summarized below. In this section and the next we will repeatedly use the fact that if bounded operators $A$ and $B$ are such that both $A B$ and $B A$ are trace class, then $\operatorname{Tr}(A B)=\operatorname{Tr}(B A)$ (cf.[27, Corollary 3.8]).

Proposition 3.4. $T^{-1} B$. Then

(1) Let $B$ be a bounded operator satisfying $B T=$

$$
B(\log T)=-(\log T) B
$$

(2) Suppose in addition that $B$ is an involution that commutes with $Q$. Then $\operatorname{Det}_{Q} T=1$.

(3) Suppose $T(\varepsilon)$ is a differentiable family of positive elliptic self-adjoint pseudo-differential operators of order zero. If $d T(\varepsilon) / d \varepsilon$ is trace class, then

$$
\frac{d}{d \varepsilon} \log \operatorname{Det}_{Q} T(\varepsilon)=\operatorname{Tr}\left(T(\varepsilon)^{-1} \frac{d T(\varepsilon)}{d \varepsilon}\right)
$$


Proof. For (1) note that

$$
\frac{1}{2 \pi \mathrm{i}} \int_{\gamma} \frac{d z}{z}(\log z)(z-T)^{-1}=(\log T) T^{-1}
$$

Indeed, from $z^{-1}(z-T)^{-1}=(z-T)^{-1} T^{-1}-z^{-1} T^{-1}$ we have

$$
\begin{aligned}
\frac{1}{2 \pi \mathrm{i}} \int_{\gamma} \frac{d z}{z}(\log z)(z-T)^{-1}= & \frac{1}{2 \pi \mathrm{i}} \int_{\gamma} d z(\log z)(z-T)^{-1} T^{-1} \\
& -\frac{1}{2 \pi \mathrm{i}} \int_{\gamma} \frac{d z}{z}(\log z) T^{-1}
\end{aligned}
$$

Because of the choice of contour, the second term vanishes. Now

$$
B(z-T)=\left(z-T^{-1}\right) B \Longrightarrow\left(z-T^{-1}\right)^{-1} B=B(z-T)^{-1}
$$

Hence,

$$
\begin{aligned}
B(\log T) & =\frac{1}{2 \pi \mathrm{i}} \int_{\gamma} d z(\log z)\left(z-T^{-1}\right)^{-1} B=\frac{1}{2 \pi \mathrm{i}} \int_{\gamma} d z(\log z)(T z-I)^{-1} T B \\
& =-\frac{1}{2 \pi \mathrm{i}} \int_{\gamma} \frac{d z}{z}(\log z)\left(z^{-1}-T\right)^{-1} T B .
\end{aligned}
$$

Next make a change of variables $w=z^{-1}$. Without loss of generality, we may assume $\gamma$ is invariant under this change. Then by (3.10).

$$
\begin{aligned}
B(\log T) & =-\frac{1}{2 \pi \mathrm{i}} \int_{\gamma} \frac{d z}{z}(\log z)\left(z^{-1}-T\right)^{-1} T B \\
& =-\frac{1}{2 \pi \mathrm{i}} \int_{\gamma} \frac{d w}{w}(\log w)(w-T)^{-1} T B=-(\log T) B
\end{aligned}
$$

For (2), it follows from (1) that

$$
\begin{aligned}
\left.f p \operatorname{Tr}\left(Q^{-s} \log T\right)\right|_{s=0} & =\left.f p \operatorname{Tr}\left(B Q^{-s}(\log T) B\right)\right|_{s=0} \\
& =\left.f p \operatorname{Tr}\left(Q^{-s} B(\log T) B\right)\right|_{s=0} \\
& =-\left.f p \operatorname{Tr}\left(Q^{-s} \log T\right)\right|_{s=0}
\end{aligned}
$$


To prove (3) we have

$$
\begin{aligned}
\frac{d}{d \varepsilon} \log T(\varepsilon)= & \frac{1}{2 \pi \mathrm{i}} \int_{\gamma} d z \log z(z-T(\varepsilon))^{-1} \frac{d T(\varepsilon)}{d \varepsilon}(z-T)^{-1} \\
\frac{d}{d \varepsilon} \log \operatorname{Det}_{Q} T(\varepsilon)= & \left.f p\right|_{s=0} \frac{1}{2 \pi \mathrm{i}} \int_{\gamma} d z(\log z) \operatorname{Tr}\left(Q^{-s}(z-T(\varepsilon))^{-1}\right. \\
& \left.\times \frac{d T(\varepsilon)}{d \varepsilon}(z-T(\varepsilon))^{-1}\right) \\
= & \frac{1}{2 \pi \mathrm{i}} \int_{\gamma} d z(\log z) \operatorname{Tr}\left((z-T(\varepsilon))^{-2} \frac{d T(\varepsilon)}{d \varepsilon}\right) \\
= & \frac{-1}{2 \pi \mathrm{i}} \int_{\gamma} d z(\log z) \frac{d}{d z} \operatorname{Tr}\left((z-T(\varepsilon))^{-1} \frac{d T(\varepsilon)}{d \varepsilon}\right) \\
= & \frac{1}{2 \pi \mathrm{i}} \int_{\gamma} \frac{d z}{z} \operatorname{Tr}\left((z-T(\varepsilon))^{-1} \frac{d T(\varepsilon)}{d \varepsilon}\right) \\
= & \operatorname{Tr}\left(T(\varepsilon)^{-1} \frac{d T(\varepsilon)}{d \varepsilon}\right) .
\end{aligned}
$$

\subsection{The Burghelea-Friedlander-Kappeler formula}

Continue to assume $M$ is closed with a collection of disjoint simple closed embedded curves $\Gamma$. We apply the definition of determinant in the previous section to the Neumann jump operator. Let $Q$ be a positive self-adjoint elliptic pseudo-differential operator $Q$ of order 1 on $\Omega_{\mathbb{R}}^{0}\left(\Gamma, \imath^{*} L\right)^{\prime \prime}$. We use the Hodge star to extend it as $\operatorname{diag}(Q, * Q *)$ on $\mathfrak{B}^{\prime \prime}\left(\Gamma, \imath^{*} L\right)$, which we continue to denote by $Q$. The self-adjoint operator $\mathcal{N}_{\Gamma}(\lambda)$ has nonzero real eigenvalues for $\lambda \neq 0$, but is not positive. Hence, we define the logarithm and determinant by

$$
\begin{aligned}
\log \mathcal{N}_{\Gamma}(\lambda) & =\frac{1}{2} \log \left(\mathcal{N}_{\Gamma}(\lambda)\right)^{2} \\
\log \operatorname{Det}_{Q} \mathcal{N}_{\Gamma}(\lambda) & =\frac{1}{2} \log \operatorname{Det}_{Q}\left(\mathcal{N}_{\Gamma}(\lambda)\right)^{2}
\end{aligned}
$$

In what follows, let $\zeta_{Q}(s)=\operatorname{Tr} Q^{-s}$, and recall that $s=0$ is a regular value of (the analytic continuation of) $\zeta_{Q}(s)$.

With this understood, we state the key factorization theorem (cf. [10, Theorem A]). 
Theorem 3.1 (BFK formula). For all $\lambda>0$,

$$
\left[\operatorname{Det}\left(D_{L}+\lambda\right)\right]_{M}=c_{Q}\left[\operatorname{Det}\left(D_{L}^{A}+\lambda\right)\right]_{M_{\Gamma}} \operatorname{Det}_{Q} \mathcal{N}_{\Gamma}(\lambda)
$$

where $c_{Q}=2^{-\zeta_{Q}(0)}$.

The rest of this section is devoted to the proof of this result. First, note the following:

Lemma 3.4. Let $\pi_{1}, \pi_{2}$ be the orthogonal projections onto the first and second factors of $\mathfrak{B}^{\prime \prime}\left(\Gamma, \imath^{*} L\right)$, and set $\dot{\mathcal{N}}_{\Gamma}(\lambda)=d \mathcal{N}_{\Gamma}(\lambda) / d \lambda$, and similarly for the operators $A$ and $R$. Then for all $\lambda>0, \pi_{i} \mathcal{N}_{\Gamma}^{-1} \dot{\mathcal{N}}_{\Gamma} \pi_{i}$ are of order -2 , and hence of trace class, for $i=1,2$. Moreover,

$$
\operatorname{Tr}\left(\pi_{1} \mathcal{N}_{\Gamma}^{-1} \dot{\mathcal{N}}_{\Gamma} \pi_{1}+\pi_{2} \mathcal{N}_{\Gamma}^{-1} \dot{\mathcal{N}}_{\Gamma} \pi_{2}\right)=\operatorname{Tr}\left((I+R(\lambda))^{-1} \dot{R}(\lambda)\right)
$$

Proof. By Proposition 3.2, $\dot{R}(\lambda)$ has order at most -2 on the circle, so the operator on the right-hand side of (3.11) is indeed trace class. In terms of the expression from Corollary 3.2,

$$
\mathcal{N}_{\Gamma}(\lambda)^{-1} \dot{\mathcal{N}}_{\Gamma}(\lambda)=A(\lambda)^{-1}(I+R(\lambda))^{-1} \dot{R}(\lambda) A(\lambda)+A(\lambda)^{-1} \dot{A}(\lambda)
$$

It therefore sufficient to prove that the operators $\pi_{i} A(\lambda)^{-1} \dot{A}(\lambda) \pi_{i}, i=1,2$, are trace class with opposite traces. But from (3.6) we have

$$
A(\lambda)^{-1} \dot{A}(\lambda)=\frac{1}{2}\left(\square_{\Gamma}+\lambda\right)^{-1}\left(\begin{array}{cc}
1 & 0 \\
-2 \nabla_{\Gamma} & -1
\end{array}\right) .
$$

The diagonal terms have order -2 on the circle and so are trace class with opposite traces, and the result follows.

The next result shows that in the special case of the Neumann jump operator the dependence of the determinant on the regularizer $Q$ is mild.

Lemma 3.5. The following hold for $\lambda$ sufficiently large:

$$
\begin{aligned}
\log \operatorname{Det}_{Q} \mathcal{N}_{\Gamma}(\lambda) & =\zeta_{Q}(0) \log 2+\int_{0}^{1} d \varepsilon \operatorname{Tr}\left((I+\varepsilon R(\lambda))^{-1} R(\lambda)\right), \\
\frac{d}{d \lambda} \log \operatorname{Det}_{Q} \mathcal{N}_{\Gamma}(\lambda) & =\operatorname{Tr}\left((I+R(\lambda))^{-1} \dot{R}(\lambda)\right)
\end{aligned}
$$


Proof. From Corollary 3.2 and the definition (3.9),

$$
\log \operatorname{Det}_{Q} \mathcal{N}_{\Gamma}(\lambda)=\zeta_{Q}(0) \log 2+\log \operatorname{Det}_{Q}((I+R(\lambda)) A(\lambda))
$$

On the other hand, $R(\lambda) A(\lambda)$ has order -2 and so is trace class. Note also that from Corollary 3.2(2), $\|R(\lambda)\|=O\left(\lambda^{-1}\right)$, so $I+\varepsilon R(\lambda)$ is uniformly invertible for $0 \leq \varepsilon \leq 1$ and $\lambda$ sufficiently large. Now applying Proposition $3.4(3)$ to the family

$$
T(\varepsilon)=((I+\varepsilon R(\lambda)) A(\lambda))^{2}
$$

and integrating the derivative in $\varepsilon$, we have

$\log \operatorname{Det}_{Q}((I+R(\lambda)) A(\lambda))=\log \operatorname{Det}_{Q} A(\lambda)+\int_{0}^{1} d \varepsilon \operatorname{Tr}\left((I+\varepsilon R(\lambda))^{-1} R(\lambda)\right)$.

Hence, (3.12) follows from (3.14) and (3.15) if we can show $\log \operatorname{Det}_{Q} A(\lambda)=$ 0. Using Corollary 3.2 and Proposition 3.4(1), $\star \log A(\lambda)=-(\log A(\lambda)) \star$, where $\star$ is given by (3.5). Since $Q$ is a diagonal operator, $\star Q=Q \star$, and the claim follows from Proposition 3.4(2). To prove (3.13), differentiate (3.12) to find

$$
\begin{aligned}
\frac{d}{d \lambda} \log \operatorname{Det}_{Q} \mathcal{N}_{\Gamma}(\lambda) & =\int_{0}^{1} d \varepsilon \operatorname{Tr}\left((I+\varepsilon R)^{-1} \dot{R}-\varepsilon(I+\varepsilon R)^{-1} \dot{R}(I+\varepsilon R)^{-1} R\right) \\
& =\int_{0}^{1} d \varepsilon \operatorname{Tr}\left((I+\varepsilon R)^{-1} \dot{R}-\varepsilon(I+\varepsilon R)^{-1} R(I+\varepsilon R)^{-1} \dot{R}\right) \\
& =\int_{0}^{1} d \varepsilon \frac{d}{d \varepsilon} \operatorname{Tr}\left(\varepsilon(I+\varepsilon R)^{-1} \dot{R}\right) \\
& =\operatorname{Tr}\left((I+R)^{-1} \dot{R}\right) .
\end{aligned}
$$

Proof of Theorem 3.1. Let $D_{L}(\lambda)=D_{L}+\lambda, D_{L}^{A}(\lambda)=D_{L}^{A}+\lambda$ and $\mathcal{P}_{M_{\Gamma}}(\lambda)$, $\mathcal{N}_{\Gamma}(\lambda)$ the associated Poisson and Neumann jump operators. By the same calculation as in [10, Corollary 3.8 and Lemma 3.6], we have

$$
\begin{aligned}
\mathcal{N}_{\Gamma}^{-1} \dot{\mathcal{N}}_{\Gamma} & =\mathbf{b}_{\Gamma}^{\prime \prime} D_{L}^{-1} \mathcal{P}_{M_{\Gamma}} \imath_{\Delta}, \\
\frac{d}{d \lambda}\left(\log \operatorname{Det} D_{L}-\log \operatorname{Det} D_{L}^{A}\right) & =\operatorname{Tr}\left(\mathcal{P}_{M_{\Gamma}} \imath_{\Delta} \mathbf{b}_{\Gamma}^{\prime \prime} D_{L}^{-1}\right),
\end{aligned}
$$

where we have omitted the spectral parameter from the notation. For simplicity, set $P=\mathcal{P}_{M_{\Gamma}} \imath_{\Delta}$ and $B=\mathbf{b}_{\Gamma}^{\prime \prime} D_{L}^{-1}$. According to [10, Lemma 3.9], $P B$ is trace class. Let $f(s)=\operatorname{Tr}\left(D_{L}^{-s} P B\right)$, which is holomorphic for $\mathfrak{R e} s>0$. 
We claim that $f$ admits an analytic continuation for $\mathfrak{R e} s>-1 / 2$. Indeed, for $\mathfrak{R e} s>0$,

$$
f(s)=\operatorname{Tr}\left(D_{L}^{-s} P B\right)=\operatorname{Tr}\left(B D_{L}^{-s} P\right)=\operatorname{Tr}\left(\pi_{1} B D_{L}^{-s} P \pi_{1}\right)+\operatorname{Tr}\left(\pi_{2} B D_{L}^{-s} P \pi_{2}\right) .
$$

But the operators $\pi_{i} B D_{L}^{-s} P \pi_{i}$ are manifested to order $-2-2 s$ on $L^{2}\left(S^{1}\right)$; hence, the claim follows. Moreover, by (3.11) and (3.13) we have $f(0)=$ $(d / d \lambda) \log \operatorname{Det}_{Q} \mathcal{N}_{\Gamma}(\lambda)$ for $\lambda$ sufficiently large. On the other hand, since $P B$ is trace class it is also true that $f(0)=\operatorname{Tr}(P B)$, and we conclude from (3.17) that

$$
\frac{d}{d \lambda} \log \operatorname{Det}_{Q} \mathcal{N}_{\Gamma}(\lambda)=\frac{d}{d \lambda}\left(\log \operatorname{Det} D_{L}-\log \operatorname{Det} D_{L}^{A}\right)
$$

for $\lambda$ large. Since the determinants are analytic in $\lambda$, this proves the existence of the constant $c_{Q}$ (alternatively, notice that (3.13) holds for all $\lambda>0$ by choosing an appropriate contour for the integral in (3.12)). The constant $c_{Q}$ may now be determined by the asymptotics as $\lambda \rightarrow \infty$. By [10, Theorem 3.12(2)], the claimed value for $c_{Q}$ holds if we show that the second term on the right-hand side of (3.12) vanishes as $\lambda \rightarrow \infty$. To see this is indeed the case, set $S(\lambda)=\square_{\Gamma}+\lambda$ acting on $\mathfrak{B}^{\prime \prime}\left(\Gamma, \imath^{*} L\right)$. It then follows from Proposition 3.2 that $S(\lambda) R(\lambda)$ is of order zero, and so it is bounded uniformly in $\lambda$ (cf.[26, Corollary 9.1]). Now $R(\lambda)=S(\lambda)^{-1}(S(\lambda) R(\lambda)$ ), so $R(\lambda)$ is trace class with $\operatorname{Tr}|R(\lambda)| \leq C \operatorname{Tr} S(\lambda)^{-1}$. The eigenvalues $\left\{\lambda_{n}\right\}_{n=1}^{\infty}$ of $S(\lambda)$ have asymptotics $\lambda_{n} \geq a n^{2}+\lambda-b$, for a positive constant $a$ and $n$ sufficiently large, and so by an explicit estimate $\operatorname{Tr} S(\lambda)^{-1}$ is $O\left(\lambda^{-1 / 2}\right)$. By the remark in the proof of Lemma 3.5, $I+\varepsilon R(\lambda)$ is uniformly invertible for $0 \leq \varepsilon \leq 1$ and $\lambda$ sufficiently large. Hence,

$$
\left|\operatorname{Tr}\left((I+\varepsilon R(\lambda))^{-1} R(\lambda)\right)\right| \leq C \operatorname{Tr}|R(\lambda)|=O\left(\lambda^{-1 / 2}\right)
$$

uniformly for $0 \leq \varepsilon \leq 1$, and the result follows.

\subsection{The case of zero modes}

The goal of this section is to extend the formula in Theorem 3.1 as $\lambda \rightarrow 0$. We will need a preliminary definition.

Definition 3.3. A framing $\tau_{L}$ near $\Gamma$ is generic if $\mathbf{b}_{\Gamma}^{\prime \prime}$ is injective on $\operatorname{ker} D_{L}$ $\subset \Omega_{\mathbb{R}}^{0}(M, L)$.

Note that an equivalent condition to the one above is that the difference map $\delta_{\Gamma} \mathbf{b}_{\partial M_{\Gamma}}^{\prime}$ be injective on $\operatorname{ker} D_{L}^{A}$ on $M_{\Gamma}$. Indeed, if $\Phi$ is a global 
section in $\operatorname{ker} D_{L}$, then regarded as a section on $M_{\Gamma}$, we automatically have $\delta_{\Gamma} \mathbf{b}_{\partial M_{\Gamma}}^{\prime}(\Phi)=0$. If in addition, $\mathbf{b}_{\Gamma}^{\prime \prime}(\Phi)=\left(\Phi^{\prime \prime}, 0\right)=0$, then $\Phi \in \operatorname{ker} D_{L}^{A}$. Conversely, if $\Phi^{A} \in \operatorname{ker} D_{L}^{A}$ and $\delta_{\Gamma} \mathbf{b}_{\partial M_{\Gamma}}^{\prime}\left(\Phi^{A}\right)=0$, then since $\delta_{\Gamma} \mathbf{b}_{\partial M_{\Gamma}}^{\prime \prime}\left(\Phi^{A}\right)=0$ automatically, it extends to a global section on $M$.

Theorem 3.2. For a given framing $\tau_{L}$ near $\Gamma$, let $\left\{\Phi_{i}\right\}$ (resp. $\left.\left\{\Phi_{i}^{A}\right\}\right)$ be a basis for $\operatorname{ker} D_{L}$ on $M$ (resp. for $\operatorname{ker} D_{L}^{A}$ on $\left.M_{\Gamma}\right)$. Assume the framing is generic in the sense of Definition 3.3. Then

$$
\left[\frac{\operatorname{Det}^{*} D_{L}}{\operatorname{det}\left(\Phi_{i}, \Phi_{j}\right)}\right]_{M}=c_{Q}\left[\frac{\operatorname{Det}^{*} D_{L}^{A}}{\operatorname{det}\left(\Phi_{i}^{A}, \Phi_{j}^{A}\right)}\right]_{M_{\Gamma}} \frac{\operatorname{det}\left(\delta_{\Gamma} \Phi_{i}^{A}, \delta_{\Gamma} \Phi_{j}^{A}\right)_{\Gamma}}{\operatorname{det}\left(\Phi_{i}^{\prime \prime}, \Phi_{j}^{\prime \prime}\right)_{\Gamma}} \operatorname{Det}_{Q}^{*} \mathcal{N}_{\Gamma}
$$

where $\mathcal{N}_{\Gamma}=\mathcal{N}_{\Gamma}(0)$ is the operator defined on the orthogonal complement of $\mathbb{A}_{\Gamma}$ in Proposition 3.3.

Proof. We apply Theorem 3.1 as $\lambda \downarrow 0$. By the definition of zeta regularization,

$$
\begin{aligned}
& \log \operatorname{Det}\left(D_{L}+\lambda\right)=(\log \lambda) \operatorname{dim}_{\mathbb{R}} \operatorname{ker} D_{L}+\log \text { Det }^{*} D_{L}+o(1), \\
& \log \operatorname{Det}\left(D_{L}^{A}+\lambda\right)=(\log \lambda) \operatorname{dim}_{\mathbb{R}} \operatorname{ker} D_{L}^{A}+\log \operatorname{Det}^{*} D_{L}^{A}+o(1)
\end{aligned}
$$

on $M$ and $M_{\Gamma}$ with Alvarez boundary conditions. Let $m=\operatorname{dim}_{\mathbb{R}} \operatorname{ker} D_{L}$ on $M$, and $n=\operatorname{dim}_{\mathbb{R}} \operatorname{ker} D_{L}^{A}$ on $M_{\Gamma}$. Hence, it is sufficient to compute $\lim _{\lambda \rightarrow 0}$ $\left\{\log \operatorname{Det}_{Q} \mathcal{N}_{\Gamma}(\lambda)+(n-m) \log \lambda\right\}$. The key point is that there are small eigenvalues of $\mathcal{N}_{\Gamma}(\lambda), \mu_{j}(\lambda) \rightarrow 0, j=1, \ldots, m$, corresponding to global holomorphic sections of $L$, and large eigenvalues $\nu_{j}(\lambda) \rightarrow+\infty, j=1, \ldots, n$, corresponding to $\operatorname{ker} D_{L}^{A}$. Moreover, it follows easily from the definition that

$$
\begin{aligned}
\log \operatorname{Det} \mathcal{N}_{\Gamma}(\lambda)= & \log \left(\mu_{1}(\lambda) \cdots \mu_{m}(\lambda)\right)+\log \left(\nu_{1}(\lambda) \cdots \nu_{n}(\lambda)\right) \\
& +\log \operatorname{Det}_{Q}^{*} \mathcal{N}_{\Gamma}+o(1) .
\end{aligned}
$$

We need therefore to compute the contribution from both the $\left\{\mu_{i}\right\}$ and $\left\{\nu_{i}\right\}$.

Let $\mu_{1}(\lambda), \ldots, \mu_{m}(\lambda)$ be the small eigenvalues of $\mathcal{N}_{\Gamma}(\lambda)$, and let $\left\{\beta_{j}(\lambda)\right\}_{j=1}^{m}$ be orthonormal with eigenvalues $\mu_{j}(\lambda)$. Let $\left\{\Phi_{j}\right\}_{i=1}^{\infty}$ be a complete set of eigensections for $D_{L}$ on $M$ with eigenvalues $\left\{\lambda_{j}\right\}_{j=1}^{\infty}, \lambda_{j}=0$ if and only if $j \leq m$. Let $\pi: \mathfrak{B}^{\prime \prime}\left(\Gamma, \imath^{*} L\right) \rightarrow \mathfrak{B}^{\prime \prime}\left(\Gamma, \imath^{*} L\right)$ orthogonal projection to $\mathbb{A}_{\Gamma}^{\mathrm{ker}}$. Then we compute

$$
\mathcal{N}_{\Gamma}^{-1}(\lambda)=\left(\begin{array}{cc}
\frac{1}{\lambda} A_{1}+\pi B_{1}(\lambda) \pi & \pi B_{1}(\lambda) \pi^{\perp} \\
\pi^{\perp} B_{1}(\lambda) \pi & \pi^{\perp} B_{1}(\lambda) \pi^{\perp}
\end{array}\right)
$$


where $A_{1}, B_{1}(\lambda): L^{2}(\Gamma) \rightarrow L^{2}(\Gamma)$ are given by

$$
\begin{aligned}
A_{1}(F, G) & =\sum_{j=1}^{m}\left((F, G), \mathbf{b}_{\Gamma}^{\prime \prime}\left(\Phi_{j}\right)\right)_{\Gamma} \mathbf{b}_{\Gamma}^{\prime \prime}\left(\Phi_{j}\right) \\
B_{1}(\lambda)(F, G) & =\sum_{j=m+1}^{\infty} \frac{1}{\lambda_{j}+\lambda}\left((F, G), \mathbf{b}_{\Gamma}^{\prime \prime}\left(\Phi_{j}\right)\right) \mathbf{b}_{\Gamma}^{\prime \prime}\left(\Phi_{j}\right) .
\end{aligned}
$$

To see this, let $\Phi$ be a section of $L \rightarrow M_{\Gamma},\left(D_{L}+\lambda\right) \Phi=0$, with $(F, G)=$ $\delta_{\Gamma} J \mathbf{b}_{\partial M_{\Gamma}}^{\prime}(\Phi)$, and $\delta_{\Gamma} \mathbf{b}_{\partial M_{\Gamma}}^{\prime \prime}(\Phi)=0$. Then by $(2.13)$,

$$
\begin{aligned}
\Phi=\sum_{j=1}^{\infty}\left(\Phi, \Phi_{j}\right)_{M_{\Gamma}} \Phi_{j} & =\sum_{j=1}^{\infty} \frac{1}{\lambda_{j}+\lambda}\left(\Phi,\left(D_{L}+\lambda\right) \Phi_{j}\right)_{M_{\Gamma}} \Phi_{j} \\
& =-\sum_{j=1}^{\infty} \frac{1}{\lambda_{j}+\lambda}\left(\mathbf{b}_{\partial M_{\Gamma}} \Phi, J \mathbf{b}_{\partial M_{\Gamma}} \Phi_{j}\right) \Phi_{j} \\
& =-\sum_{j=1}^{\infty} \frac{1}{\lambda_{j}+\lambda}\left(\delta_{\Gamma} \mathbf{b}_{\Gamma} \Phi, J \mathbf{b}_{\Gamma} \Phi_{j}\right) \Phi_{j} \\
& =-\sum_{j=1}^{\infty} \frac{1}{\lambda_{j}+\lambda}\left(\delta_{\Gamma} \mathbf{b}_{\Gamma}^{\prime} \Phi, J \mathbf{b}_{\Gamma}^{\prime \prime} \Phi_{j}\right) \Phi_{j} \\
& =\sum_{j=1}^{\infty} \frac{1}{\lambda_{j}+\lambda}\left(\delta_{\Gamma} J \mathbf{b}_{\Gamma}^{\prime} \Phi, \mathbf{b}_{\Gamma}^{\prime \prime} \Phi_{j}\right) \Phi_{j} \\
& =\sum_{j=1}^{\infty} \frac{1}{\lambda_{j}+\lambda}\left((F, G), \mathbf{b}_{\Gamma}^{\prime \prime} \Phi_{j}\right) \Phi_{j}
\end{aligned}
$$

and computing $\mathbf{b}_{\Gamma}^{\prime \prime}(\Phi)$ gives the result. We wish to relate the eigenvalues of $A_{1}$ to the $\mu_{j}(\lambda)$. Since

$$
\mathcal{N}_{\Gamma}^{-1}(\lambda) \beta_{j}(\lambda)=\mu_{j}^{-1}(\lambda) \beta_{j}(\lambda)
$$

we have

$$
\begin{aligned}
\frac{1}{\lambda} A_{1} \beta_{j}(\lambda)+\pi B_{1}(\lambda) \beta_{j}(\lambda) & =\mu_{j}^{-1}(\lambda) \pi \beta_{j}(\lambda) \\
\pi^{\perp} B_{1}(\lambda) \beta_{j}(\lambda) & =\mu_{j}^{-1}(\lambda) \pi^{\perp} \beta_{j}(\lambda) .
\end{aligned}
$$

Since $B_{1}(\lambda)$ is uniformly bounded as $\lambda \downarrow 0$, it follows that $\left\|\pi^{\perp} \beta_{j}(\lambda)\right\|_{L^{2}(\Gamma)} \leq$ $C \mu_{j}(\lambda)$, for $C$ independent of $\lambda$. In particular, $\left\|\pi \beta_{j}(\lambda)\right\|_{L^{2}(\Gamma)} \rightarrow 1$ as $\lambda \downarrow 0$, 
and so (after passing to a sequence $\lambda_{k} \downarrow 0$ ) there exist limits $\left\{\beta_{j}(0)\right\}$ which give a basis for $\mathbb{A}_{\Gamma}^{\mathrm{ker}}$. If we let $v_{j}$ be an orthonormal basis for $\mathbb{A}_{\Gamma}^{\mathrm{ker}}$ such that $A_{1} v_{j}=\sigma_{j} v_{j}$, and write

$$
\pi \beta_{j}(\lambda)=\sum_{k=1}^{m} C_{j k}(\lambda) v_{k}
$$

then the (subsequential) limit $C_{j k}(0)$ exists and is nonsingular. From (3.20) we have

$$
\left\|A_{1} \pi \beta_{j}(\lambda)-\frac{\lambda}{\mu_{j}(\lambda)} \pi \beta_{j}(\lambda)\right\|_{L^{2}(\Gamma)} \leq C \lambda
$$

In terms of the basis $\left\{v_{j}\right\}$,

$$
A_{1} \pi \beta_{j}(\lambda)-\frac{\lambda}{\mu_{j}(\lambda)} \pi \beta_{j}(\lambda)=\sum_{k=1}^{m} C_{j k}(\lambda)\left(\sigma_{k}-\frac{\lambda}{\mu_{j}(\lambda)}\right) v_{k}
$$

so by $(3.21), C_{j k}(\lambda)\left(\sigma_{k}-\left(\lambda / \mu_{j}(\lambda)\right)\right) \rightarrow 0$, for all $j, k$. Since $\left(C_{j k}\right)$ is nonsingular, for each $j, C_{j k}(0) \neq 0$ for some $k$. Hence, $\sigma_{k}^{-1}=\lim _{\lambda \downarrow 0} \mu_{j}(\lambda) / \lambda=\hat{\mu}_{j}$ exists for each $j$, with $C_{j k} \sigma_{k}=\hat{\mu}_{j}^{-1} C_{j k}$. Again using the fact that $\left(C_{j k}\right)$ is nonsingular, we have

$$
\log \operatorname{det} A_{1}+\log \left(\prod \mu_{j}(\lambda)\right)=m \log \lambda+o(1)
$$

Finally, note that by choosing $\mathbf{b}_{\Gamma}^{\prime \prime}\left(\Phi_{j}\right), j=1, \ldots, m$, as a basis in $(3.19)$, we have

$$
\begin{gathered}
\operatorname{det} A_{1}=\operatorname{det}\left(\mathbf{b}_{\Gamma}^{\prime \prime}\left(\Phi_{i}\right), \mathbf{b}_{\Gamma}^{\prime \prime}\left(\Phi_{j}\right)\right) \\
\log \left(\prod \mu_{j}(\lambda)\right)=m \log \lambda-\log \operatorname{det}\left(\mathbf{b}_{\Gamma}^{\prime \prime}\left(\Phi_{i}\right), \mathbf{b}_{\Gamma}^{\prime \prime}\left(\Phi_{j}\right)\right)+o(1)
\end{gathered}
$$

Let $\nu_{1}(\lambda), \ldots, \nu_{n}(\lambda)$ be the divergent eigenvalues of $\mathcal{N}_{\Gamma}(\lambda)$, and let $\left\{\beta_{j}^{A}(\lambda)\right\}_{j=1}^{n}$ be orthonormal with eigenvalues $\nu_{j}(\lambda)$. Let $\left\{\Phi_{i}^{A}\right\}_{i=1}^{\infty}$ be a complete set of eigensections for $D_{L}^{A}$ on $M_{\Gamma}$ with eigenvalues $\left\{\lambda_{i}\right\}_{i=1}^{\infty}$, and $\lambda_{i}=0$ if and only if $i \leq n$. Let $\pi: \mathfrak{B}^{\prime \prime}\left(\Gamma, \imath^{*} L\right) \rightarrow \mathfrak{B}^{\prime \prime}\left(\Gamma, \imath^{*} L\right)$ be orthogonal projection to $\mathbb{A}_{\Gamma}^{\text {alv }}$. We also choose a smooth extension map $E: \mathfrak{B}^{\prime \prime}\left(\Gamma, \imath^{*} L\right) \rightarrow L^{2}\left(M_{\Gamma}\right)$ satisfying $\mathbf{b}_{\Gamma}^{\prime \prime} E=I, \mathbf{b}_{\Gamma}^{\prime} E=0$. Then as above we 
compute

$$
\mathcal{N}_{\Gamma}(\lambda)=\left(\begin{array}{cc}
\frac{1}{\lambda} A_{2}(\lambda)+\pi B_{2}(\lambda) \pi & \pi B_{2}(\lambda) \pi^{\perp} \\
\pi^{\perp} B_{2}(\lambda) \pi & \pi^{\perp} B_{2}(\lambda) \pi^{\perp}
\end{array}\right)
$$

where $A_{2}(\lambda), B_{2}(\lambda): L^{2}(\Gamma) \rightarrow L^{2}(\Gamma)$ are given by

$$
\begin{aligned}
A_{2}(\lambda)(f, g)= & -\sum_{j=1}^{n}\left\{\left((f, g), \delta_{\Gamma} J \mathbf{b}_{\partial M_{\Gamma}}^{\prime}\left(\Phi_{j}^{A}\right)\right)\right. \\
& \left.+\lambda\left(E(f, g), \Phi_{j}^{A}\right)_{M}\right\} \delta_{\Gamma} J \mathbf{b}_{\partial M_{\Gamma}}^{\prime}\left(\Phi_{i}^{A}\right), \\
B_{2}(\lambda)(f, g)= & -\sum_{j=n+1}^{\infty} \frac{1}{\lambda_{j}+\lambda}\left(\left(D_{L}+\lambda\right) E(f, g), \Phi_{j}^{A}\right)_{M} \delta_{\Gamma} J \mathbf{b}_{\partial M_{\Gamma}}^{\prime}\left(\Phi_{j}^{A}\right) .
\end{aligned}
$$

To see this, note that to compute $\mathcal{N}_{\Gamma}(\lambda)(f, g)$ we need to solve the boundary value problem

$$
\left(D_{L}+\lambda\right) \Phi=0, \quad \mathbf{b}_{\partial M_{\Gamma}}^{\prime \prime}(\Phi)=\imath_{\Delta}(f, g)
$$

on $M_{\Gamma}$. From the definition of the extension, it is sufficient to solve

$$
\left(D_{L}+\lambda\right) \widetilde{\Phi}=-\left(D_{L}+\lambda\right) E(f, g), \quad \mathbf{b}_{\partial M_{\Gamma}}^{\prime \prime}(\widetilde{\Phi})=0
$$

for $\Phi=E(f, g)+\widetilde{\Phi}$, and by the assumption on $E$ the jump in $\mathbf{b}_{\partial M_{\Gamma}^{\prime}}(\widetilde{\Phi})$ gives $\mathcal{N}_{\Gamma}(\lambda)(f, g)$. Now

$$
\begin{aligned}
\widetilde{\Phi}= & -\sum_{j=1}^{\infty} \frac{1}{\lambda_{j}+\lambda}\left(\left(D_{L}+\lambda\right) E(f, g), \Phi_{j}^{A}\right)_{M_{\Gamma}} \Phi_{j}^{A} \\
= & -\sum_{j=1}^{n}\left\{\frac{1}{\lambda}\left(D_{L} E(f, g), \Phi_{j}^{A}\right)_{M_{\Gamma}}+\left(E, \Phi_{j}\right)_{M_{\Gamma}}\right\} \Phi_{j}^{A} \\
& -\sum_{j=n+1}^{\infty} \frac{1}{\lambda_{j}+\lambda}\left(\left(D_{L}+\lambda\right) E(f, g), \Phi_{j}^{A}\right)_{M_{\Gamma}} \Phi_{j}^{A} .
\end{aligned}
$$


By $(2.13)$, the first term on the right-hand side is (since $\mathbf{b}_{\partial M_{\Gamma}^{\prime \prime}}\left(\Phi_{j}^{A}\right)=0$ )

$$
\begin{aligned}
& =-\sum_{j=1}^{n}\left\{\frac{1}{\lambda}\left(\mathbf{b}_{\partial M_{\Gamma}}(E(f, g)), J \mathbf{b}_{\partial M_{\Gamma}}\left(\Phi_{j}^{A}\right)\right)+\left(E(f, g), \Phi_{j}\right)_{M_{\Gamma}}\right\} \Phi_{j}^{A} \\
& =-\sum_{j=1}^{n}\left\{\frac{1}{\lambda}\left(\mathbf{b}_{\partial M_{\Gamma}}^{\prime \prime}(E(f, g)), J \mathbf{b}_{\partial M_{\Gamma}}^{\prime}\left(\Phi_{j}^{A}\right)\right)+\left(E(f, g), \Phi_{j}^{A}\right)_{M_{\Gamma}}\right\} \Phi_{j}^{A} \\
& =-\sum_{j=1}^{n}\left\{\frac{1}{\lambda}\left(\mathbf{b}_{\Gamma}^{\prime \prime}(E(f, g)), \delta_{\Gamma} J \mathbf{b}_{\partial M_{\Gamma}}^{\prime}\left(\Phi_{j}^{A}\right)\right)+\left(E(f, g), \Phi_{j}^{A}\right)_{M_{\Gamma}}\right\} \Phi_{j}^{A} \\
& =-\sum_{j=1}^{n}\left\{\frac{1}{\lambda}\left((f, g), \delta_{\Gamma} J \mathbf{b}_{\partial M_{\Gamma}}^{\prime}\left(\Phi_{j}^{A}\right)\right)+\left(E(f, g), \Phi_{j}^{A}\right)_{M_{\Gamma}}\right\} \Phi_{j}^{A} .
\end{aligned}
$$

We again relate the eigenvalues of $A_{2}(0)$ to the $\nu_{j}(\lambda)$. Since $\mathcal{N}_{\Gamma}(\lambda) \beta_{j}^{A}(\lambda)=$ $\nu_{j}(\lambda) \beta_{j}^{A}(\lambda)$, we have

$$
\begin{aligned}
\frac{1}{\lambda} A_{2}(\lambda) \beta_{j}^{A}(\lambda)+\pi B_{2}(\lambda) \beta_{j}^{A}(\lambda) & =\nu_{j}(\lambda) \pi \beta_{j}^{A}(\lambda), \\
\pi^{\perp} B_{2}(\lambda) \beta_{j}^{A}(\lambda) & =\nu_{j}(\lambda) \pi^{\perp} \beta_{j}^{A}(\lambda) .
\end{aligned}
$$

Since $B_{2}(\lambda)$ is uniformly bounded as $\lambda \downarrow 0$, it follows that $\left\|\pi^{\perp} \beta_{j}^{A}(\lambda)\right\|_{L^{2}(\Gamma)} \leq$ $C \nu_{j}^{-1}(\lambda)$, for $C$ independent of $\lambda$. In particular, $\left\|\pi \beta_{j}^{A}(\lambda)\right\|_{L^{2}(\Gamma)} \rightarrow 1$ as $\lambda \downarrow 0$, and so the (sequential) limits $\left\{\beta_{j}^{A}(0)\right\}$ give a basis for $\mathbb{A}_{\Gamma}^{\text {alv }}$. If we let $v_{j}$ be an orthonormal basis for $\mathbb{A}_{\Gamma}^{\text {alv }}$ such that $A_{2}(0) v_{j}=\sigma_{j} v_{j}$, and write

$$
\pi \beta_{j}^{A}(\lambda)=\sum_{k=1}^{n} C_{j k}(\lambda) v_{k}
$$

then $C_{j k}(0)$ exists and is nonsingular. From (3.24) we have

$$
\left\|A_{2}(0) \pi \beta_{j}^{A}(\lambda)-\lambda \nu_{j}(\lambda) \pi \beta_{j}^{A}(\lambda)\right\|_{L^{2}(\Gamma)} \leq C \lambda .
$$

In terms of the basis $\left\{v_{j}\right\}$,

$$
A_{2}(0) \pi \beta_{j}^{A}(\lambda)-\lambda \nu_{j}(\lambda) \pi \beta_{j}^{A}(\lambda)=\sum_{k=1}^{n} C_{j k}(\lambda)\left(\sigma_{k}-\lambda \nu_{j}(\lambda)\right) v_{k}
$$

so by $(3.25), C_{j k}(\lambda)\left(\sigma_{k}-\lambda \nu_{j}(\lambda)\right) \rightarrow 0$ for all $j, k$. As before, $\lim _{\lambda \downarrow 0} \lambda \nu_{j}(\lambda)=$ $\hat{\nu}_{j}$ exists for each $j$, and $C_{j k} \sigma_{k}=\hat{\nu}_{j} C_{j k}$ for all $j, k$. Hence, $\log \operatorname{det} A_{2}(0)=$ $\log \left(\prod \nu_{j}(\lambda)\right)+m \log \lambda+o(1)$. Finally, note that by choosing $\delta_{\Gamma} J \mathbf{b}_{\partial M_{\Gamma}}^{\prime}\left(\Phi_{j}^{A}\right)$ 
as a basis in (3.23), we have

$$
\log \left(\prod \nu_{j}(\lambda)\right)=-m \log \lambda+\log \operatorname{det}\left(\delta_{\Gamma} \mathbf{b}_{\partial M_{\Gamma}}^{\prime}\left(\Phi_{i}^{A}\right), \delta_{\Gamma} \mathbf{b}_{\partial M_{\Gamma}}^{\prime}\left(\Phi_{j}^{A}\right)\right)+o(1) .
$$

Putting together (3.18), (3.22) and (3.26) gives the result.

We will later use the following special case of Theorem 3.2: let $\Gamma$ be a simple closed connected curve separating $M$ into components $R^{(1)}$ and $R^{(2)}$. Then for any choice of bases $\left\{\Phi_{i}\right\}_{i=1}^{m}$ for $\operatorname{ker} D_{L}$ on $M$, and $\left\{\Phi_{i}^{A,(1)}\right\}_{i=1}^{m_{1}}$, and $\left\{\Phi_{i}^{A,(2)}\right\}_{i=1}^{m_{2}}$ for $\operatorname{ker} D_{L}^{A}$ on $R^{(1)}$ and $R^{(2)}$, we have

$$
\begin{aligned}
{\left[\frac{\operatorname{Det}^{*} D_{L}}{\operatorname{det}\left(\Phi_{i}, \Phi_{j}\right)}\right]_{M}=} & c_{Q}\left[\frac{\operatorname{Det}^{*} D_{L}^{A}}{\operatorname{det}\left(\Phi_{i}^{A,(1)}, \Phi_{j}^{A,(1)}\right)}\right]_{R^{(1)}}\left[\frac{\operatorname{Det}^{*} D_{L}^{A}}{\operatorname{det}\left(\Phi_{i}^{A,(2)}, \Phi_{j}^{A,(2)}\right)}\right]_{R^{(2)}} \\
& \times \frac{\operatorname{det}\left(\Phi_{i}^{A}, \Phi_{j}^{A}\right)_{\Gamma}}{\operatorname{det}\left(\Phi_{i}^{\prime \prime}, \Phi_{j}^{\prime \prime}\right)_{\Gamma}} \operatorname{Det}_{Q}^{*} \mathcal{N}_{\Gamma}
\end{aligned}
$$

where

$$
\Phi_{i}^{A}= \begin{cases}\Phi_{i}^{A,(1)}, & 1 \leq i \leq m_{1} \\ \Phi_{i-m_{1}}^{A,(2)}, & m_{1}<i \leq m_{1}+m_{2}\end{cases}
$$

extended by zero to the whole surface $M_{\Gamma}$.

Actually, for the purpose of degeneration it will be useful to also have a slightly modified version of (3.27) in the case where the trivialization $\tau_{L}$ is, in fact, the restriction of a global holomorphic section. This is not a generic situation in the sense of Definition 3.3, since the global section $\tau_{L}$ also satisfies Alvarez boundary conditions, and hence $\operatorname{det}\left(\Phi_{i}^{\prime \prime}, \Phi_{j}^{\prime \prime}\right)_{\Gamma}=0$ for any basis. Similarly, since the jump of $\tau_{L}$ is trivial, $\operatorname{det}\left(\delta_{\Gamma} \Phi_{i}^{A}, \delta_{\Gamma} \Phi_{j}^{A}\right)$ also vanishes. This motivates the following:

Definition 3.4. Let $\tau_{L}$ be a global holomorphic section of $L \rightarrow M$, nowhere vanishing near $\Gamma$. We call the framing $\tau_{L}$ good if the kernel of $\mathbf{b}_{\Gamma}^{\prime \prime}$ on $\operatorname{ker} D_{L} \subset$ $\Omega_{\mathbb{R}}^{0}(M, L)$ is precisely the $\mathbb{R}$-span of $\tau_{L}$. We say that bases $\left\{\Phi_{i}\right\}_{i=1}^{m}$, $\left\{\Phi_{i}^{A,(1)}\right\}_{i=1}^{m_{1}}$, and $\left\{\Phi_{i}^{A,(2)}\right\}_{i=1}^{m_{2}}$ for $\operatorname{ker} D_{L}$ on $M$ and for $\operatorname{ker} D_{L}^{A}$ on $R^{(1)}$ and $R^{(2)}$, are adapted to $\tau_{L}$ if $\Phi_{1}=\tau_{L}, \Phi_{1}^{A,(1)}=\left.\tau_{L}\right|_{R^{(1)}}, \Phi_{1}^{A,(2)}=\left.\tau_{L}\right|_{R^{(2)}}$. 
For adapted bases the notation $\operatorname{det}^{*}\left(\Phi_{i}, \Phi_{j}\right)_{\Gamma}$ will by definition denote the determinant of the (11)-minor of $\left(\Phi_{i}, \Phi_{j}\right)_{\Gamma}$. Similarly for $\Phi_{i}^{A}$. Then after some linear algebra we have

Theorem 3.3. Let $\tau_{L}$ be a global holomorphic section giving a framing of $L$ near a simple closed separating curve $\Gamma$, and let $\left\{\Phi_{i}\right\}\left(\operatorname{resp} .\left\{\Phi_{i}^{A,(1)}, \Phi_{j}^{A,(2)}\right\}\right)$ be an adapted basis for $\operatorname{ker} D_{L}$ on $M$ (resp. on $R^{(1,2)}$ with Alvarez boundary conditions). Assume the framing is good in the sense of Definition 3.4. Then

$$
\begin{aligned}
{\left[\frac{\operatorname{Det}^{*} D_{L}}{\operatorname{det}\left(\Phi_{i}, \Phi_{j}\right)}\right]_{M}=} & c_{Q}\left[\frac{\operatorname{Det}^{*} D_{L}^{A}}{\operatorname{det}\left(\Phi_{i}^{A,(1)}, \Phi_{j}^{A,(1)}\right)}\right]_{R^{(1)}}\left[\frac{\operatorname{Det}^{*} D_{L}^{A}}{\operatorname{det}\left(\Phi_{i}^{A,(2)}, \Phi_{j}^{A,(2)}\right)}\right]_{R^{(2)}} \\
& \times \frac{\operatorname{det}^{*}\left(\Phi_{i}^{A}, \Phi_{j}^{A}\right)_{\Gamma}}{\operatorname{det}^{*}\left(\Phi_{i}^{\prime \prime}, \Phi_{j}^{\prime \prime}\right)_{\Gamma}} \operatorname{Det}_{Q}^{*} \mathcal{N}_{\Gamma}
\end{aligned}
$$

Example 3.2. As a special case of Theorem 3.2, consider the two-sphere $S_{R}^{2}$ of radius $R$ cut along an equator $\Gamma$ into two copies of the hemisphere $H_{R}^{2}$. Choose the canonical bundle $K$ with the canonical framing. Then ker $D_{L}$ and ker $D_{L}^{A}$ are both trivial, so the condition in Definition 3.3 is trivially satisfied. Moreover, it is easy to see that $c_{Q}$ Det $\mathcal{N}_{\Gamma}=1$. Using this and Remark 2.4 (4),

$$
\left[\operatorname{Det}^{*} D_{\mathcal{O}}\right]_{S_{R}^{2}}=\left[\operatorname{Det} D_{K}\right]_{S_{R}^{2}}=c_{Q}\left[\operatorname{Det} D_{K}^{A}\right]_{H_{R}^{2}}^{2} \operatorname{Det} \mathcal{N}_{\Gamma}=\left[\operatorname{Det}^{*} D_{\mathcal{O}}^{A}\right]_{H_{R}^{2}}^{2}
$$

and so by Lemma 2.2 and Remark 2.4(3), we obtain the well-known formula:

$$
\left[\operatorname{Det}^{*} \Delta\right]_{S_{R}^{2}}=\left[\operatorname{Det}_{\text {neu. }}^{*} \Delta\right]_{H_{R}^{2}}\left[\operatorname{Det}_{\text {dir. }} \Delta\right]_{H_{R}^{2}}
$$

\section{Asymptotics of determinants}

\subsection{Asymptotics of the generalized Neumann jump operator}

The goal of this section is to prove the following. Let $M$ be a closed Riemann surface of genus $g$, and choose a coordinate neighborhood $B$ with coordinate $z$ centered at $p \in M$. Let $B_{\varepsilon}=\{|z|<\varepsilon\}$, and set $R_{\varepsilon}=M \backslash B_{\varepsilon}$. Let $L \rightarrow M$ be a hermitian holomorphic line bundle of degree $d$ with a global holomorphic section $\tau_{L}$ that is nowhere vanishing on $B$. Also, assume coker $P_{L}=\{0\}$ on $M$ and on $R_{\varepsilon}$, and that $\rho \equiv 1$ and $\left\|\tau_{L}\right\|=1$ on $B$. 
Proposition 4.1. If $\mathcal{N}_{\Gamma_{\varepsilon}}$ denotes the Neumann jump operator with respect to Alvarez boundary conditions defined by a global section $\tau_{L}$. Then as $\varepsilon \rightarrow 0$,

$$
\log \operatorname{Det}_{Q}^{*} \mathcal{N}_{\Gamma_{\varepsilon}} \longrightarrow\left(\zeta_{Q}(0)-4 h^{0}(L)+2\right) \log 2 .
$$

By direct computation, as in [33] one proves

Lemma 4.1. For $1 / 2 \geq \varepsilon>0, \mathcal{A}_{R_{\varepsilon}}=S_{\varepsilon}+\varepsilon U_{\varepsilon} \mathcal{A}_{R_{1}}\left(I+T_{\varepsilon} \mathcal{A}_{R_{1}}\right)^{-1} U_{\varepsilon}$, where

$$
\begin{aligned}
& S_{\varepsilon}(f, g)(\theta)=\sum_{n \neq 0}\left(\frac{\varepsilon^{n}-\varepsilon^{-n}}{\varepsilon^{n}+\varepsilon^{-n}}\right)\left(\begin{array}{cc}
0 & -\mathrm{i} \\
\mathrm{i} & -\varepsilon / n
\end{array}\right)\left(\begin{array}{l}
\hat{f}(n) \\
\hat{g}(n)
\end{array}\right) \mathrm{e}^{\mathrm{i} n \theta}, \\
& U_{\varepsilon}(f, g)(\theta)=\sum_{n \neq 0} \frac{2}{\varepsilon\left(\varepsilon^{n}+\varepsilon^{-n}\right)}\left(\begin{array}{c}
\hat{f}(n) \\
\varepsilon \hat{g}(n)
\end{array}\right) \mathrm{e}^{\mathrm{i} n \theta}, \\
& T_{\varepsilon}(f, g)(\theta)=\sum_{n \neq 0}\left(\frac{\varepsilon^{n}-\varepsilon^{-n}}{\varepsilon^{n}+\varepsilon^{-n}}\right)\left(\begin{array}{cc}
1 / n & -\mathrm{i} \\
\mathrm{i} & 0
\end{array}\right)\left(\begin{array}{l}
\hat{f}(n) \\
\hat{g}(n)
\end{array}\right) \mathrm{e}^{\mathrm{i} n \theta}
\end{aligned}
$$

for functions $f, g$ in (3.3).

We also note the following estimates.

Lemma 4.2. Then assume $1 / 2 \geq \varepsilon>0$.

(1) $\left(A_{\varepsilon}-S_{\varepsilon}\right)$ is trace class with norm bounded by $8 \varepsilon^{2}$.

(2) $U_{\varepsilon}$ is trace class with uniformly bounded norm.

(3) If $T_{0}$ is defined by

$$
T_{0}(f, g)(\theta)=\sum_{n \neq 0}\left(\begin{array}{cc}
-1 /|n| & \mathrm{i} \sigma(n) \\
-\mathrm{i} \sigma(n) & 0
\end{array}\right)\left(\begin{array}{l}
\hat{f}(n) \\
\hat{g}(n)
\end{array}\right) \mathrm{e}^{\mathrm{i} n \theta},
$$

then $\left(T_{\varepsilon}-T_{0}\right)$ is trace class with norm bounded by $8 \varepsilon^{2}$.

Lemma 4.3. For $\varepsilon>0$ sufficiently small, $I+T_{\varepsilon} \mathcal{A}_{R_{1}}$ is uniformly invertible on the orthogonal complement of $\mathbb{A}_{\Gamma}$.

Proof. It suffices to show that $I+T_{0} \mathcal{A}_{R_{1}}$ has no kernel on $\mathbb{A}_{\Gamma}^{\perp}$. But by a direct computation, if $(f, g)=-T_{0} \mathcal{A}_{R_{1}}(f, g)$, then $\mathcal{P}_{R_{1}}(f, g)$ extends to a global section in ker $D_{L}$. 
Proof of Proposition 4.1. By Lemma 4.1 we have on the orthogonal complement of $\mathbb{A}_{\Gamma_{\varepsilon}}$ :

$$
\begin{aligned}
\log \mathcal{N}_{\Gamma_{\varepsilon}} & =\log 2+\frac{1}{2} \log \left(\frac{1}{2} \mathcal{N}_{\Gamma_{\varepsilon}}\right)^{2}=\log 2+\frac{1}{2} \log (I+C(\varepsilon)), \\
\log \operatorname{Det}_{Q}^{*} \mathcal{N}_{\Gamma_{\varepsilon}} & =\left(\zeta_{Q}(0)-\operatorname{dim}_{\mathbb{R}} \mathbb{A}_{\Gamma_{\varepsilon}}\right) \log 2+\frac{1}{2} \log \operatorname{Det}_{Q}^{*}(I+C(\varepsilon)) .
\end{aligned}
$$

More precisely, assume the orientation of $\Gamma$ is chosen to agree with $\partial R_{\varepsilon}$, and let $f, g$ be functions as in (3.3). Let $\Sigma$ be the involution that sends $\hat{f}(n) \mapsto \hat{f}(-n)$ and $\hat{g}(n) \mapsto \hat{g}(-n)$. Now using (3.2),

$$
\begin{aligned}
\mathcal{N}_{\Gamma_{\varepsilon}}= & \mathcal{A}_{R_{\varepsilon}}+\Sigma \circ \mathcal{A}_{B_{\varepsilon}} \circ \Sigma \\
\mathcal{N}_{\Gamma_{\varepsilon}}\left(\begin{array}{l}
f \\
g
\end{array}\right)= & \sum_{n \neq 0}\left[\left(\begin{array}{cc}
0 & \mathrm{i} \sigma(n) \\
-\mathrm{i} \sigma(n) & \varepsilon /|n|
\end{array}\right)+\Sigma \circ\left(\begin{array}{cc}
0 & -\mathrm{i} \sigma(n) \\
\mathrm{i} \sigma(n) & -\varepsilon /|n|
\end{array}\right) \circ \Sigma\right]\left(\begin{array}{l}
\hat{f}(n) \\
\hat{g}(n)
\end{array}\right) \mathrm{e}^{\mathrm{i} n \theta} \\
& +\{\operatorname{trace} \operatorname{class}\} \\
= & \sum_{n \neq 0} 2\left(\begin{array}{c}
\mathrm{i} \sigma(n) \hat{g}(n) \\
-\mathrm{i} \sigma(n) \hat{f}(n)
\end{array}\right) \mathrm{e}^{\mathrm{i} n \theta+\{\text { trace class }\}} \\
\mathcal{N}_{\Gamma_{\varepsilon}}^{2}= & 4 I+\{\operatorname{trace} \text { class }\} .
\end{aligned}
$$

Now by Lemma 3.3,

$$
\begin{aligned}
\operatorname{dim}_{\mathbb{R}} \mathbb{A}_{\Gamma_{\varepsilon}} & =\operatorname{dim}_{\mathbb{R}} \operatorname{ker} D_{L}-1+\operatorname{dim}_{\mathbb{R}} \operatorname{ker} D_{L}^{A}-1=2 \operatorname{dim}_{\mathbb{R}} \operatorname{ker} D_{L}-2 \\
& =4 h^{0}(L)-2 .
\end{aligned}
$$

Since $C(\varepsilon) \rightarrow 0$ in trace, the result follows from Proposition 3.4(3).

Next, we assume $L$ has a framing given by a global meromorphic section with simple pole at $p$. It is easy to see that for an appropriate annular coordinate on the disk $L$ is isomorphic as a framed bundle to the canonical bundle with canonical framing. In this case we have the following asymptotics.

Proposition 4.2. If $\mathcal{N}_{\Gamma_{\varepsilon}}$ denotes the Neumann jump operator with respect to Alvarez boundary conditions defined by a global meromorphic section $\tau_{L}$ with simple pole at $p$, then as $\varepsilon \rightarrow 0$,

$$
\log \operatorname{Det}_{Q}^{*} \mathcal{N}_{\Gamma_{\varepsilon}}+\log (\varepsilon / 2) \longrightarrow\left(\zeta_{Q}(0)-4 h^{0}(L)-2\right) \log 2 .
$$

Proof. The computation is nearly identical to the one above, except now $\operatorname{dim}_{\mathbb{R}} \mathbb{A}_{\Gamma_{\varepsilon}}=4 h^{0}(L)+1$, and the constant mode $(1,0) \notin \mathbb{A}_{\Gamma_{\varepsilon}}^{\mathrm{ker}}$. By assumption, $\mathcal{A}_{R_{\varepsilon}}(1,0)=(0,0)$, and by direct computation for the canonical bundle 
on the disk, $\mathcal{A}_{B_{\varepsilon}}(1,0)=(2 / \varepsilon, 0)$. Factoring this out from the determinant, the result follows.

\subsection{Admissible metrics and asymptotics of $S(\sigma, f)$}

Recall the definition of the Arakelov metric (cf. [3, 14, 15, 32]). Given a compact Riemann surface $M$ of genus $g \geq 1$, let $\left\{A_{i}, B_{i}\right\}_{i=1}^{g}$ be a symplectic set of generators of $H_{1}(M)$ and choose $\left\{\omega_{i}\right\}_{i=1}^{g}$ to be a basis of abelian differentials normalized such that $\int_{A_{i}} \omega_{j}=\delta_{i j}$. Let $\Omega_{i j}=\int_{B_{i}} \omega_{j}$ be the associated period matrix with theta function $\vartheta$. Set

$$
\mu=\frac{i}{2 g} \sum_{i, j=1}^{g}(\mathfrak{I m} \Omega)_{i j}^{-1} \omega_{i} \wedge \bar{\omega}_{j}
$$

Then $\int_{M} \mu=1$. The Arakelov-Green's function $G(z, w)$ is symmetric with a zero of order one along the diagonal satisfying $\partial \bar{\partial} \log G(z, w)=(\pi \mathrm{i}) \mu$, for $z \neq w$, normalized by

$$
\int_{M} \mu(z) \log G(z, w)=0 .
$$

The Arakelov metric $\rho_{A r}=\rho_{A r}(z)|d z|^{2}$ is defined by

$$
\log \rho_{A r}(z)=2 \lim _{w \rightarrow z}\{\log G(z, w)-\log |z-w|\}
$$

A hermitian metric $h$ on a line bundle $L \rightarrow M$ of degree is $d$ is admissible in the sense of [14] if

$$
\operatorname{Ric}(h)=-(2 \pi \mathrm{i} d) \mu
$$

The Arakelov metric on $M$, considered as a hermitian metric on the anticanonical bundle $K^{*}$, is admissible:

$$
\operatorname{Ric}\left(\rho_{A r}\right)=4 \pi \mathrm{i}(g-1) \mu \text {. }
$$

In terms of the Hermitian-Einstein tensor and the scalar curvature, (4.3) and (4.4) become

$$
\begin{aligned}
d A \Omega_{L, h} & =(2 \pi d) \mu, \\
d A R_{\rho_{A r}} & =-8 \pi(g-1) \mu .
\end{aligned}
$$

For more details we refer to the papers cited above. 
We now return to the situation in the previous section. Let $R_{\varepsilon}=M \backslash B_{\varepsilon}$, where $B_{\varepsilon}$ is the coordinate neighborhood $|z|<\varepsilon$ centered at a point $p$. Let $L \rightarrow M$ be a holomorphic line bundle with admissible metric $h$, and let $L(p)=L \otimes \mathcal{O}(p)$. Choosing an admissible metric on $\mathcal{O}(p)$ gives an admissible metric on $L(p)$. Let $\hat{\omega}_{0}$ be a global holomorphic section of $L(p)$ that is nonvanishing at $p$, and let $\mathbb{1}_{p}$ be a global holomorphic section of $\mathcal{O}(p)$ vanishing at $p$. Using the framings given by $\hat{\omega}_{0} \otimes \mathbb{1}_{p}^{-1}$ and $\hat{\omega}_{0}$, respectively, then on $R_{\varepsilon}, L$ and $L(p)$ are naturally isomorphic as framed bundles, and their hermitian metrics are conformal with factor $f(z)=-\log G(z, p)$. With this understood, we have the following simple computation.

Lemma 4.4. Let $S_{\varepsilon}(f)=S(0, f)$ denote the Liouville action (2.18) on $R_{\varepsilon}$. Then $S_{\varepsilon}(f) \rightarrow 0$ as $\varepsilon \rightarrow 0$.

Proof. Note that the local expression for the metric in the framing on $L(p)$ is continuous as $\varepsilon \rightarrow 0$. Hence, if we let $\hat{h}$ denote the metric on $L(p)$ and $h$ that on $L$, then by (2.18),

$$
\begin{aligned}
S_{\varepsilon}(f)= & -\frac{1}{\pi} \int_{M_{\varepsilon}} d A_{\rho}|\nabla f|^{2}-\frac{1}{2 \pi} \int_{M_{\varepsilon}} d A_{\rho}\left(4 \Omega_{L(p), \hat{h}}+R_{\rho}\right) f \\
& +\frac{1}{\pi} \int_{\partial M_{\varepsilon}} d s_{\rho}\left(2 \nu_{L(p), \hat{h}}-\kappa_{\rho}\right) f .
\end{aligned}
$$

Using (4.1), (4.5) and the remark above,

$$
\begin{aligned}
S_{\varepsilon}(f) & \simeq-\frac{1}{\pi} \int_{M_{\varepsilon}} d A_{\rho}|\nabla f|^{2}-\frac{1}{\pi} \int_{\partial M_{\varepsilon}} d s_{\rho} \kappa_{\rho} f \\
& =\frac{1}{\pi} \int_{M_{\varepsilon}} d A_{\rho} f \Delta f-\frac{1}{\pi} \int_{\partial M_{\varepsilon}} d A_{\rho} f \partial_{n} f-\frac{1}{\pi} \int_{\partial M_{\varepsilon}} d s_{\rho} \kappa_{\rho} f \\
& \simeq-\frac{1}{\pi} \int_{\partial M_{\varepsilon}} d A_{\rho} f \partial_{n} f-\frac{1}{\pi} \int_{\partial M_{\varepsilon}} d s_{\rho} \kappa_{\rho} f
\end{aligned}
$$

which vanishes as $\varepsilon \rightarrow 0$.

\subsection{Proof of Theorem 1.2}

Let $L \rightarrow M$ with $\operatorname{deg} L=d$ and $h^{1}(L)=0$, and set $L(p)=L \otimes \mathcal{O}(p)$. Set $N=h^{0}(L)=d-g+1$ and $m=2 N+1$. Let $\left\{\omega_{i}\right\}_{i=1}^{N}$ be a fixed basis for $H^{0}(M, L)$, and set $\hat{\omega}_{i}=\omega_{i} \otimes \mathbb{1}_{p}$. We assume that the framings $\hat{\omega}_{0} \otimes \mathbb{1}_{p}^{-1}$ and $\hat{\omega}_{0}$ are generic and good in the sense of Definitions 3.3 and 3.4. We will need 
technical results on degenerations of sections. The proofs of the following two lemmas are straightforward and will be omitted.

Lemma 4.5. With the assumption $h^{1}(L)=0$, $\operatorname{ker} P_{L}^{\dagger}$ (and therefore also $\left.\operatorname{ker} P_{L(p)}^{\dagger}\right)$ vanishes on $R_{\varepsilon}$ for $\varepsilon>0$ sufficiently small.

Lemma 4.6. Let $\nu_{i}$ be the order of vanishing of $\omega_{i}$ at $p$. Then for any sequence $\varepsilon_{k} \rightarrow 0$ there is a subsequence (also denoted $\left\{\varepsilon_{k}\right\}$ ) and a collection $\left\{\omega_{i, \varepsilon_{k}}\right\}_{i=1}^{m}, \omega_{m, \varepsilon_{k}}=\hat{\omega}_{0} \otimes \mathbb{1}_{p}^{-1}$ for all $k$, satisfying the following:

- The set $\left\{\omega_{i, \varepsilon_{k}}\right\}_{i=1}^{m}$ is a real basis for the subspace of ker $\bar{\partial}_{L}$ on $R_{\varepsilon_{k}}$ with $\omega_{i, \varepsilon_{k}}=f_{i, \varepsilon_{k}} \hat{\omega}_{0} \otimes \mathbb{1}_{p}^{-1} \quad$ near $\quad p \quad$ satisfying $\left.\quad \mathfrak{I m}\left(f_{i, \varepsilon_{k}}\right)\right|_{|z|=\varepsilon_{k}}=0 \quad$ (cf. Remark 2.1).

- For each $1 \leq j \leq N$,

$$
\begin{gathered}
\sup _{z \in R_{\varepsilon_{k}}} \varepsilon_{k}^{-\nu_{i}}\left|\omega_{2 j-1, \varepsilon_{k}}(z)-\omega_{j}(z)\right| \longrightarrow 0 \\
\sup _{z \in R_{\varepsilon_{k}}} \varepsilon_{k}^{-\nu_{i}}\left|\omega_{2 j, \varepsilon_{k}}(z)-\mathrm{i} \omega_{j}(z)\right| \longrightarrow 0
\end{gathered}
$$

as $k \rightarrow \infty$.

Set $\Phi_{m}=\jmath\left(\hat{\omega}_{0} \otimes \mathbb{1}_{p}^{-1}\right), \widehat{\Phi}_{m}=\jmath\left(\hat{\omega}_{0}\right)$ and $\widehat{\Phi}_{m+1}=\jmath\left(i \hat{\omega}_{0}\right)$. For $1 \leq i \leq m$, set $\Phi_{i, \varepsilon_{k}}=\jmath\left(\omega_{i, \varepsilon_{k}}\right), \widehat{\Phi}_{i, \varepsilon_{k}}=\jmath\left(\omega_{i, \varepsilon_{k}} \otimes \mathbb{1}_{p}\right)$, and for $1 \leq j \leq N$, set

$$
\begin{aligned}
\Phi_{2 j} & =\jmath\left(\mathrm{i} \omega_{j}\right), \quad \widehat{\Phi}_{2 j}=\jmath\left(\mathrm{i} \hat{\omega}_{2 j}\right), \\
\Phi_{2 j-1} & =\jmath\left(\omega_{j}\right), \quad \widehat{\Phi}_{2 j-1}=\jmath\left(\hat{\omega}_{2 j}\right) .
\end{aligned}
$$

We will need the following asymptotics for the sections chosen as above.

Lemma 4.7. Assume, without loss of generality, that the metric on $M$ is locally euclidean on a neighborhood of $p$. Then as $k \rightarrow \infty$,

$$
\begin{aligned}
& \operatorname{det}^{*}\left(\widehat{\Phi}_{i}^{\prime \prime}, \widehat{\Phi}_{j}^{\prime \prime}\right)_{\partial R_{\varepsilon_{k}}} \simeq \operatorname{det}\left(\Phi_{i}^{\prime \prime}, \Phi_{j}^{\prime \prime}\right)_{\partial R_{\varepsilon_{k}}}\left(2 \pi \varepsilon_{k}\left\|\widehat{\Phi}_{m}(p)\right\|^{2}\right)\left(\rho_{A r}(p) \varepsilon_{k}^{2}\right)^{m-1}, \\
& \operatorname{det}\left(\widehat{\Phi}_{i, \varepsilon_{k}}^{A}, \widehat{\Phi}_{j, \varepsilon_{k}}^{A}\right)_{\partial R_{\varepsilon_{k}}} \simeq \operatorname{det}\left(\Phi_{i, \varepsilon_{k}}^{A}, \Phi_{j, \varepsilon_{k}}^{A}\right)_{\partial R_{\varepsilon_{k}}}\left(\rho_{A r}(p) \varepsilon_{k}^{2}\right)^{m}
\end{aligned}
$$

(where here because of the choice of indexing, det* denotes minus the determinant of the $m \times m$ minor, unlike in Theorem 3.3). 
Proof. It suffices to prove an estimate for $\operatorname{det}\left\langle\omega_{2 i, \varepsilon_{k}}, \omega_{2 j, \varepsilon_{k}}\right\rangle_{\partial R_{\varepsilon_{k}}}$. Write the expansion

$$
f_{2 i, \varepsilon_{k}}(z)=\sum_{n \in \mathbb{Z}} a_{n, \varepsilon_{k}}^{(i)} z^{n}
$$

Note that the condition $\left.\mathfrak{I m}\left(f_{2 i, \varepsilon}\right)\right|_{|z|=\varepsilon_{k}}=0$ implies $a_{-n, \varepsilon_{k}}^{(i)}=\bar{a}_{n, \varepsilon_{k}}^{(i)} \varepsilon_{k}^{2 n}$, for $n \geq 0$. By Lemma 4.6, we have $\left|a_{\nu_{i}, \varepsilon_{k}}^{(i)}\right| \neq 0$ and $\left|\varepsilon_{k}^{n-\nu_{i}} a_{n, \varepsilon_{k}}^{(i)}\right| \rightarrow 0$, for $n \neq \nu_{i}$. Hence,

$$
\begin{aligned}
\left\langle\omega_{2 i, \varepsilon_{k}}, \omega_{2 j, \varepsilon_{k}}\right\rangle_{\partial R_{\varepsilon_{k}}} & \simeq \sum_{\ell, n} \int_{0}^{2 \pi} a_{\ell, \varepsilon_{k}}^{(i)} \bar{a}_{n, \varepsilon_{k}}^{(j)} z^{\ell} \bar{z}^{n} \varepsilon_{k} d \theta \times \frac{\left\|\hat{\omega}_{0}(p)\right\|^{2}}{\left(\rho_{A r}(p) \varepsilon_{k}^{2}\right)} \\
& \simeq 2 \pi \varepsilon_{k}^{-1} \sum_{n \in \mathbb{Z}} a_{n, \varepsilon_{k}}^{(i)} \bar{a}_{n, \varepsilon_{k}}^{(j)} \varepsilon_{k}^{2 n} \times \frac{\left\|\hat{\omega}_{0}(p)\right\|^{2}}{\rho_{A r}(p)} \\
\varepsilon_{k}^{-\nu_{i}-\nu_{j}+1}\left\langle\omega_{2 i, \varepsilon_{k}}, \omega_{2 j, \varepsilon_{k}}\right\rangle_{\partial R_{\varepsilon_{k}}} & \simeq 2 \pi \sum_{n \in \mathbb{Z}} \varepsilon_{k}^{n-\nu_{i}} a_{n, \varepsilon_{k}}^{(i)} \varepsilon_{k}^{n-\nu_{j}} \bar{a}_{n, \varepsilon_{k}}^{(j)} \times \frac{\left\|\hat{\omega}_{0}(p)\right\|^{2}}{\rho_{A r}(p)} \\
& \simeq 2 \pi\left|a_{\nu_{i}, 0}^{(i)}\right|^{2} \delta_{i j} \times \frac{\left\|\hat{\omega}_{0}(p)\right\|^{2}}{\rho_{A r}(p)}+o(1) .
\end{aligned}
$$

Similarly,

$$
\varepsilon_{k}^{-\nu_{i}-\nu_{j}-1}\left\langle\hat{\omega}_{2 i, \varepsilon_{k}}, \hat{\omega}_{2 j, \varepsilon_{k}}\right\rangle_{\partial R_{\varepsilon_{k}}} \simeq 2 \pi\left|a_{\nu_{i}, 0}^{(i)}\right|^{2} \delta_{i j} \times\left\|\hat{\omega}_{0}(p)\right\|^{2}+o(1) .
$$

The second estimate in the lemma follows from this. The proof of the first estimate is similar.

We now turn to the proof of Theorem 1.2. On the one hand, for $L$ we may apply (3.27) to get

$$
\begin{aligned}
{\left[\frac{\operatorname{Det}^{*} D_{L}}{\operatorname{det}\left(\Phi_{i}, \Phi_{j}\right)}\right]_{M}=} & c_{Q}\left[\frac{\operatorname{Det}^{*} D_{L}^{A}}{\operatorname{det}\left(\Phi_{i, \varepsilon_{k}}^{A}, \Phi_{j, \varepsilon_{k}}^{A}\right)}\right]_{R_{\varepsilon_{k}}}\left[\operatorname{Det}^{*} D_{K}^{A}\right]_{B_{\varepsilon_{k}}} \\
& \times \frac{\operatorname{det}\left(\Phi_{i, \varepsilon_{k}}^{A}, \Phi_{j, \varepsilon_{k}}^{A}\right)_{\Gamma}}{\operatorname{det}\left(\Phi_{i}^{\prime \prime}, \Phi_{j}^{\prime \prime}\right)_{\Gamma}} \operatorname{Det}_{Q}^{*} \mathcal{N}_{\Gamma} .
\end{aligned}
$$

On the other hand, from Theorem 3.3 applied to $L(p)$, we obtain

$$
\begin{aligned}
{\left[\frac{\operatorname{Det}^{*} D_{L(p)}}{\operatorname{det}\left(\widehat{\Phi}_{i}, \widehat{\Phi}_{j}\right)}\right]_{M}=} & c_{Q}\left[\frac{\operatorname{Det}^{*} D_{L(p)}^{A}}{\operatorname{det}\left(\widehat{\Phi}_{i, \varepsilon_{k}}^{A}, \widehat{\Phi}_{j, \varepsilon_{k}}^{A}\right)}\right]_{R_{\varepsilon_{k}}}\left[\frac{\operatorname{Det}^{*} D_{\mathcal{O}}^{A}}{\left\|\widehat{\Phi}_{m}\right\|^{2}}\right]_{B_{\varepsilon_{k}}} \\
& \times \frac{\operatorname{det}\left(\widehat{\Phi}_{i, \varepsilon_{k}}^{A}, \widehat{\Phi}_{j, \varepsilon_{k}}^{A}\right)_{\Gamma}}{\operatorname{det}^{*}\left(\widehat{\Phi}_{i}^{\prime \prime}, \widehat{\Phi}_{j}^{\prime \prime}\right)_{\Gamma}} \widehat{\operatorname{det}}_{Q}^{*} \widehat{\mathcal{N}}_{\Gamma} .
\end{aligned}
$$


Now on $R_{\varepsilon_{k}}$, the framed bundles $L$ and $L(p)$ are isomorphic, and by Lemma 4.4

$$
\left[\frac{\operatorname{Det}^{*} D_{L}^{A}}{\operatorname{det}\left(\Phi_{i, \varepsilon_{k}}^{A}, \Phi_{j, \varepsilon_{k}}^{A}\right)}\right]_{R_{\varepsilon_{k}}} \simeq\left[\frac{\operatorname{Det}^{*} D_{L(p)}^{A}}{\operatorname{det}\left(\widehat{\Phi}_{i, \varepsilon_{k}}^{A}, \widehat{\Phi}_{j, \varepsilon_{k}}^{A}\right)}\right]_{R_{\varepsilon_{k}}} .
$$

By Proposition 2.2, $\operatorname{Det}^{*} D_{K}^{A}=\operatorname{Det}^{*} D_{\mathcal{O}}^{A} \quad$ on the disk. Applying Propositions 4.1 and 4.2 (using Lemma 4.5 and noting that $h^{0}(L(p))=$ $\left.h^{0}(L)+1\right)$,

$$
\begin{aligned}
& c_{Q} \operatorname{Det}_{Q}^{*} \mathcal{N}_{\Gamma} \simeq\left(2 / \varepsilon_{k}\right) 2^{-4 h^{0}(L(p))+2}, \\
& c_{Q} \operatorname{Det}_{Q}^{*} \widehat{\mathcal{N}}_{\Gamma} \simeq 2^{-4 h^{0}(L(p))+2} .
\end{aligned}
$$

Hence,

$$
\begin{aligned}
{\left[\frac{\operatorname{Det}^{*} D_{L(p)}}{\operatorname{det}\left(\widehat{\Phi}_{i}, \widehat{\Phi}_{j}\right)}\right]_{M} } & \simeq \frac{\left(\varepsilon_{k} / 2\right)}{\left\|\widehat{\Phi}_{m}\right\|_{B_{\varepsilon_{k}}}^{2}} \frac{\operatorname{det}\left(\widehat{\Phi}_{i, \varepsilon_{k}}^{A}, \widehat{\Phi}_{j, \varepsilon_{k}}^{A}\right)_{\Gamma}}{\operatorname{det}\left(\widehat{\Phi}_{i}^{\prime \prime}, \widehat{\Phi}_{j}^{\prime \prime}\right)_{\Gamma}}\left[\frac{\operatorname{det}\left(\Phi_{i, \varepsilon_{k}}^{A}, \Phi_{j, \varepsilon_{k}}^{A}\right)_{\Gamma}}{\operatorname{det}\left(\Phi_{i}^{\prime \prime}, \Phi_{j}^{\prime \prime}\right)_{\Gamma}}\right]^{-1} \\
& \times\left[\frac{\operatorname{Det}^{*} D_{L}}{\operatorname{det}\left(\Phi_{i}, \Phi_{j}\right)}\right]_{M} .
\end{aligned}
$$

Finally,

$$
\left\|\widehat{\Phi}_{m}\right\|_{B_{\varepsilon_{k}}}^{2} \simeq \pi \varepsilon_{k}^{2}\left\|\Phi_{m}(p)\right\|^{2} \rho_{A r}(p)
$$

Combining this with Lemma 4.7 and letting $k \rightarrow \infty$, we have

$$
4 \pi^{2}\left\|\widehat{\Phi}_{0}\right\|^{4}(p)\left[\frac{\operatorname{Det}^{*} D_{L(p)}}{\operatorname{det}\left(\widehat{\Phi}_{i}, \widehat{\Phi}_{j}\right)}\right]_{M}=\left[\frac{\operatorname{Det}^{*} D_{L}}{\operatorname{det}\left(\Phi_{i}, \Phi_{j}\right)}\right]_{M} .
$$

The result now follows from Lemma 2.2.

\section{Acknowledgment}

The author wishes to thank E. Falbel, A. Kokotov, D.H. Phong and the referees for their suggestions. He especially thanks S. Zelditch for many discussions and in particular for pointing out ref. [17]. The hospitality of the University of Paris 6 and the IHES, where a portion of this work was completed, is also gratefully acknowledged. Research supported in part by NSF grant DMS-1037094. 


\section{References}

[1] O. Alvarez, Theory of strings with boundaries: fluctuations, topology, and quantum geometry, Nuclear Phys. B 216 (1983), $125-184$.

[2] L. Alvarez-Gaumé, G. Moore, and C. Vafa, Theta functions, modular invariance, and strings, Comm. Math. Phys. 106(1) (1986), 1-40.

[3] S. Arakelov, An intersection theory for divisors on an arithmetic surface, Math. USSR Izv. 8 (1974), 1179-1192.

[4] C. Beneventano, P. Gilkey, K. Kirsten and E. Santangelo, Strong ellipticity and spectral properties of chiral bag boundary conditions, J. Phys. A 36(45) (2003), 11533-11543.

[5] J.-M. Bismut and G. Lebeau, Complex immersions and Quillen metrics, Inst. Hautes Etudes Sci. Publ. Math. 74 (1991), ii+298 pp.

[6] J.-B. Bost, Conformal and holomorphic anomalies on Riemann surfaces and determinant line bundles. VIIIth International Congress on Mathematical Physics (Marseille, 1986), World Sci. Publishing, Singapore, 1987, 768-775.

[7] J.-B. Bost and P. Nelson, Spin-1/2 bosonization on compact surfaces, Phys. Rev. Lett. 57(7) (1986), 795-798.

[8] L. Alvarez-Gaumé, J.-B. Bost, G. Moore, P. Nelson, and C. Vafa, Bosonization on higher genus Riemann surfaces, Comm. Math. Phys. $112(3)$ (1987), 503-552.

[9] T. Branson and P. Gilkey, The asymptotics of the Laplacian on a manifold with boundary, Comm. Partial Differential Equations 15(2) (1990), 245-272.

[10] D. Burghelea, L. Friedlander and T. Kappeler, Meyer-Vietoris type formula for determinants of elliptic differential operators, J. Funct. Anal. $\mathbf{1 0 7}(1)$ (1992), 34-65.

[11] P. Deligne, Le déterminant de la cohomologie, Current trends in arithmetical algebraic geometry (Arcata, CA, 1985), Contemp. Math., 67, Amer. Math. Soc., Providence, RI, 1987, 93-177.

[12] E. D'Hoker and D.H. Phong, The geometry of string perturbation theory, Rev. Mod. Phys. 60(4) (1988), 917-1065. 
[13] M. Dugan and H. Sonoda, Functional determinants on Riemann surfaces, Nucl. Phys. B 289(1) (1987), 227-252.

[14] G. Faltings, Calculus on arithmetic surfaces, Ann. of Math. (2) 119(2) (1984), 387-424.

[15] J. Fay, Kernel functions, analytic torsion, and moduli spaces, Mem. Amer. Math. Soc. 96, No. 464 (1992), vi+123 pp.

[16] R. Forman, Functional determinants and geometry, Invent. Math. 88(3) (1987), 447-493.

[17] L. Friedlander and V. Guillemin, Determinants of zeroth order operators, J. Differential Geom. 78(1) (2008), 1-12.

[18] P. Gilkey, Invariance theory, the heat equation, and the Atiyah-Singer index theorem, 2nd ed., Studies in Advanced Math., CRC Press, Boca Raton, FL, 1995, x+516 pp.

[19] H. Gillet and C. Soulé, Analytic torsion and the arithmetic Todd genus, With an appendix by D. Zagier, Topology 30(1) (1991), 21-54.

[20] J. Jorgenson, Degenerating hyperbolic Riemann surfaces and an evaluation of the constant in Deligne's arithmetic Riemann-Roch theorem, unpublished manuscript.

[21] S. Kobayashi, Differential geometry of complex vector bundles, Publications of the Mathematical Society of Japan, 15, Kanô Memorial Lectures, 5, Princeton University Press, Princeton, NJ; Iwanami Shoten, Tokyo, 1987, xii+305 pp.

[22] J. Park, Gluing formulae of spectral invariants and Cauchy data spaces, Analysis, Geometry and Topology of Elliptic Operators, World Sci. Publ., Hackensack, NJ, 2006, 23-38.

[23] D. Quillen, Determinants of Cauchy-Riemann operators on Riemann surfaces, Funktsional. Anal. i Prilozhen. 19(1) (1985), $37-41,96$.

[24] D. Ray and I. Singer, Analytic torsion for complex manifolds, Ann. of Math. (2) 98 (1973), 154-177.

[25] R. Seeley, Complex powers of an elliptic operator, Singular Integrals (Proc. Sympos. Pure Math., Chicago, IL, 1966), Amer. Math. Soc., Providence, RI, 1967, 288-307. 
[26] M. Shubin, Pseudodifferential operators and spectral theory (translated from the 1978 Russian original by Stig I. Andersson), 2nd ed., SpringerVerlag, Berlin, 2001, xii+288 pp.

[27] B. Simon, Trace ideals and their applications, 2nd ed., Mathematical Surveys and Monographs, 120, American Mathematical Society, Providence, RI, 2005, viii+150 pp.

[28] H. Sonoda, Calculation of a propagator on a Riemann surface, Phys. Lett. B 178(4) (1986), 390-394.

[29] C. Soulé, Géométrie d'Arakelov des surfaces arithmétiques, Séminaire Bourbaki, 1988/89, Astérisque, No. 177-178, 1989, Exp. No. 713, 327-343.

[30] D. Vassilevich, Heat kernel expansion: user's manual, Phys. Rep. 388(5-6) (2003), 279-360.

[31] E. Verlinde and H. Verlinde, Chiral bosonization, determinants and the string partition function, Nucl. Phys. B 288(2) (1987), 357-396.

[32] R. Wentworth, The asymptotics of the Arakelov-Green's function and Faltings' delta invariant, Comm. Math. Phys. 137 (1991), 427-459.

[33] R. Wentworth, Precise constants in bosonization formulas I, Comm. Math. Phys. 282 (2008), 339-355.

Department of Mathematics

UNIVERSITY OF MARYLAND

College Park, MD 20742

USA

E-mail address: raw@umd.edu

Received OCtober 12, 2011 
